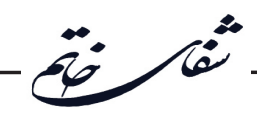

\title{
Comparison of Effectiveness of Group Schema Therapy and Group Acceptance and Commitment Therapy on Symptoms of Borderline Personality Disorder of Patients with Substance Abuse
}

\author{
Mahmood Piri ${ }^{1}$, Ali Hosseinaei ${ }^{2 *}$, Javanshir Asadi ${ }^{1}$, Kazem Shariatnia ${ }^{2}$ \\ ${ }^{1}$ Department of Psychology, Gorgan Branch, Islamic Azad University, Gorgan, Iran \\ ${ }^{2}$ Department of Psychology, Azadshar Branch, Islamic Azad University, Azadshar, Iran
}

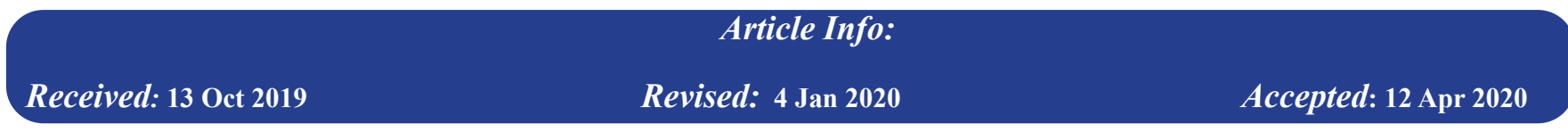

\section{ABSTRACT}

Introduction: Personality disorders are one of the most important causes of drug abuse and improvement that is crucial for the treatment of subjects with addiction. This study aimed to compare the effectiveness of group psychotherapy based on schema therapy versus acceptance and commitment therapy on symptoms of borderline personality disorder among patients with substance abuse. Materials and Methods: The study was a semi-experimental inves tigation with a pretest-posttest and follow-up and control groups. The population included all addicted residing in two addiction treatment campuses in Gonbad-e-Kavoos city, Iran, in the first half of 2018, among which a sample of 30 individuals was selected by available sampling methods which were assigned into two experimental groups and one control group randomly. Instruments were Millon Clinical Multiaxial Inventory (MCMI-III). The first experimental group received the schema therapy intervention and the second experimental group received acceptance and commitment therapy in twelve 90-minute sessions in groups The control group did not receive any intervention. A follow-up test was performed one-month after the post-test. Results: The data indicated that both therapeutic interventions were effective in reducing the symptoms of borderline personality disorders, while the control group did not change significantly. Furthermore, there was a significant difference between the effectiveness of schema therapy and acceptance and commitment therapy at the end of the treatment and in a one-month follow-up. Schema therapy was more effective in reduction of the symptoms of borderline personality disorder compared to the other groups. Conclusion: The results of this study can inspire addiction specialists to use the aforementioned psychotherapy for treatment of borderline personality disorder of substance abusers and improving their psychological ability to successfully quit and prevent their relapse.

Key words:

1. Acceptance and Commitment Therapy

2. Borderline Personality

Disorder

3. Psychotherapy

4. Substance-Related Disorders

*Corresponding Author: Ali Hosseinaei

E-mail: a.hosseinaei@gmail.com 


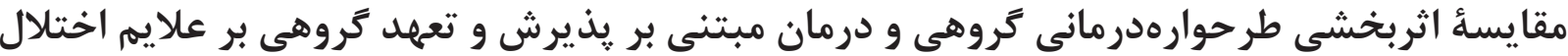 شخصيت مرزى مبتلايان به سوء مرومان مبنى برف مواد مخدر
}

\author{
محمود يِيرى'، على حسينائى ז"، جوانشير اسدى'، كاظم شريعت نيا' \\ اكروه روانشناسى، واحد كر كان، دانشعاه آزاد اسلامى، كر كان، ايران

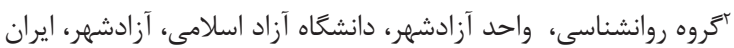

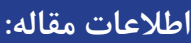

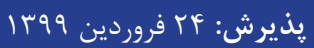

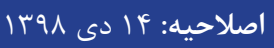

دريافت: آ مهر شوسا

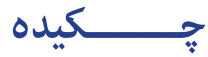

مقدمه: اختلالات شخصيت يكى از مهمترين عوامل كرايش به به سوء مصرف مواد است كه براى درمان مان

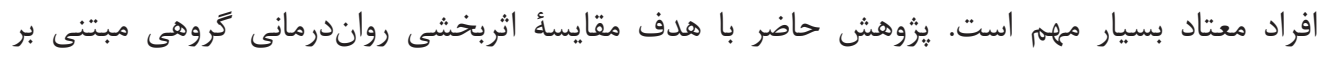

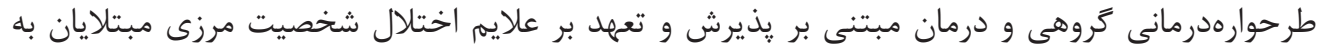

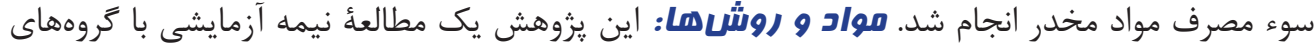

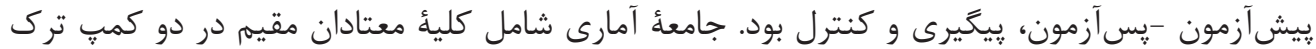

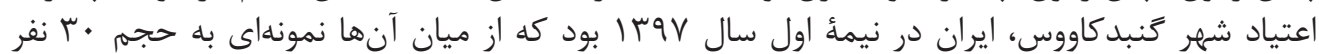

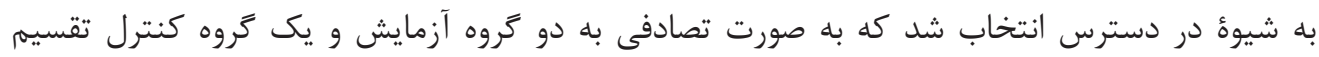

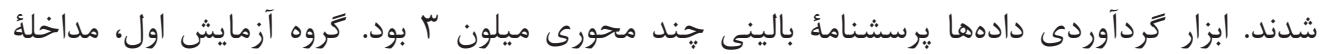

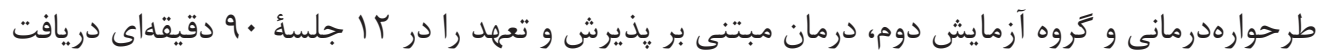

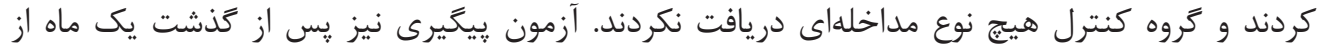

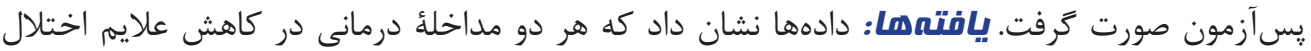

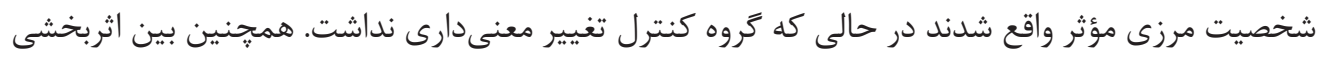

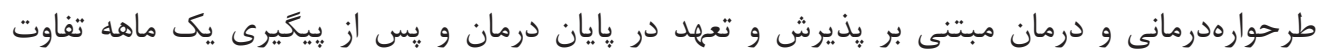

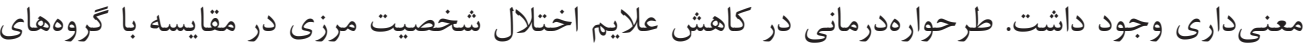

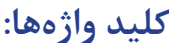

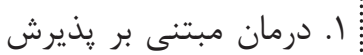
و

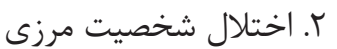

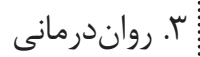

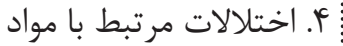

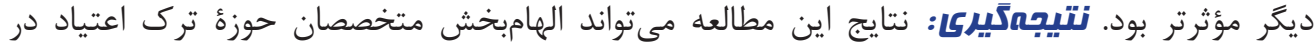

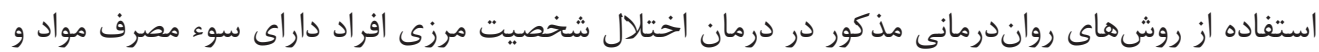

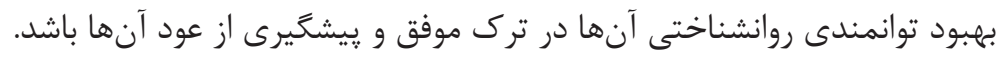

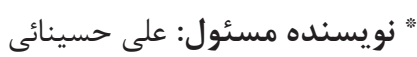
a.hosseinaei@gmail.com آدرس الكترونيكى 


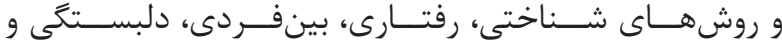

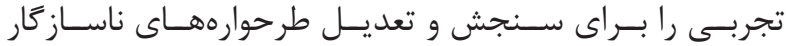

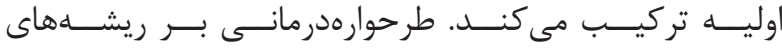

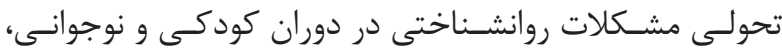

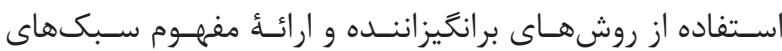

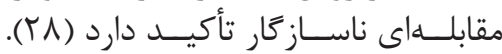

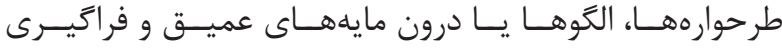

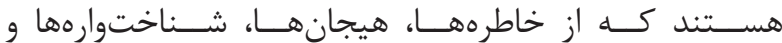

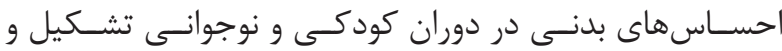

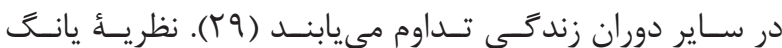

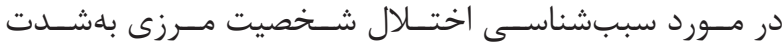

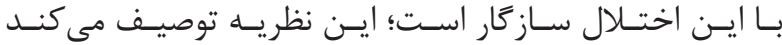

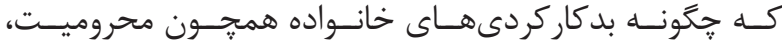

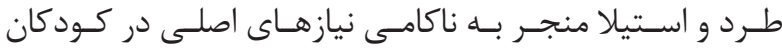

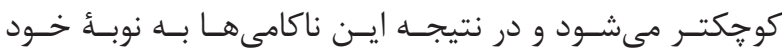

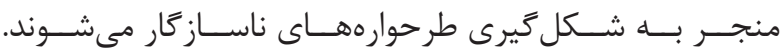

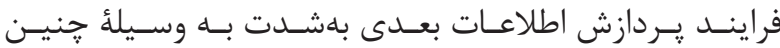

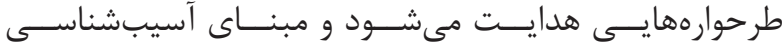

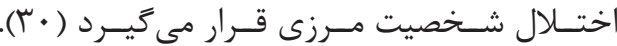

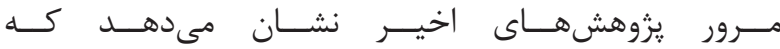

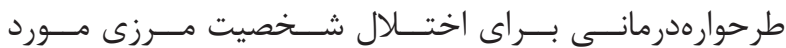

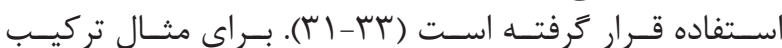

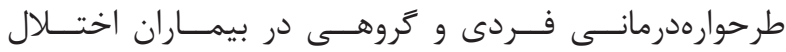

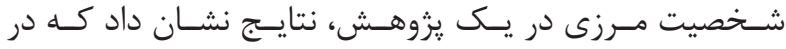

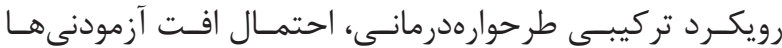

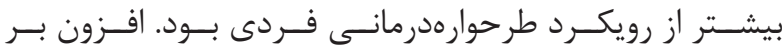

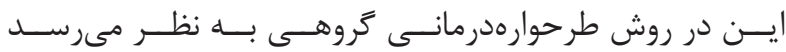

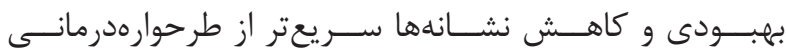

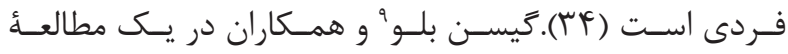

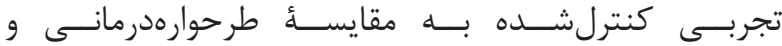

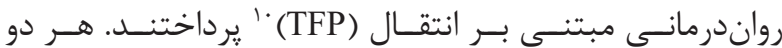

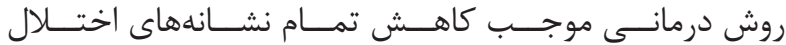

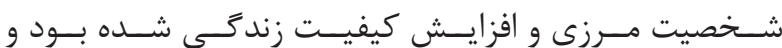

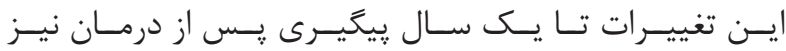

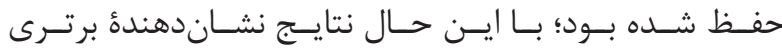

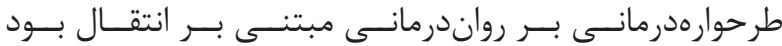

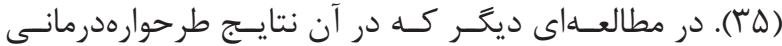

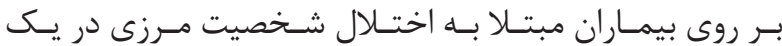

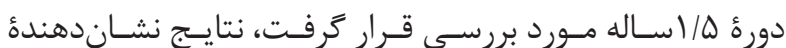

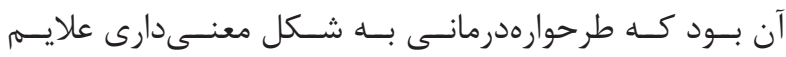

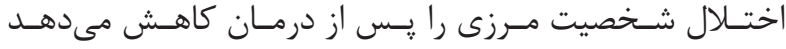

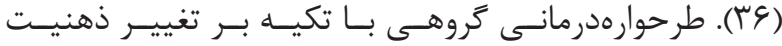

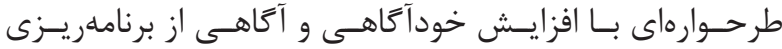

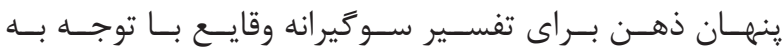

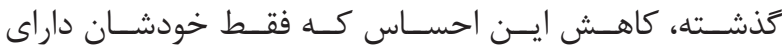

\footnotetext{
${ }^{1}$ Borderline personality disorder

${ }^{2}$ Schema therapy

${ }^{3}$ Young

${ }^{4}$ Gestalt

${ }^{5}$ Object relations
}

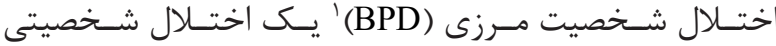

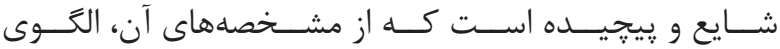

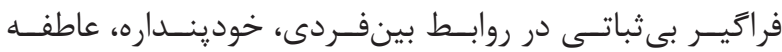

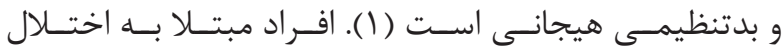

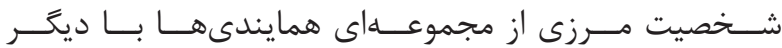

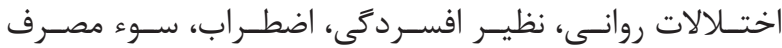

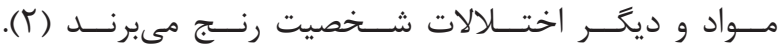

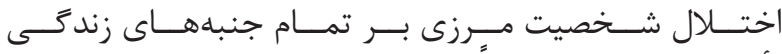

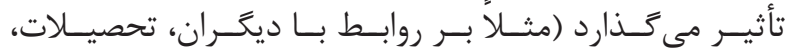

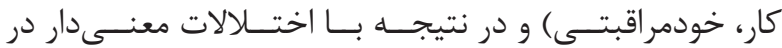

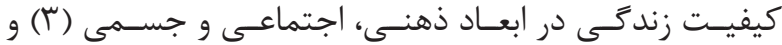

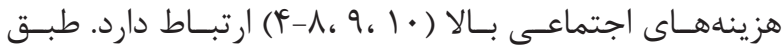

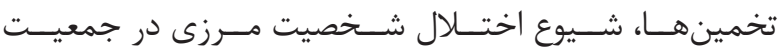

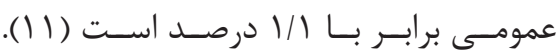

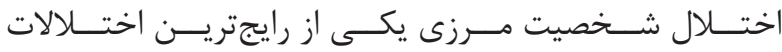

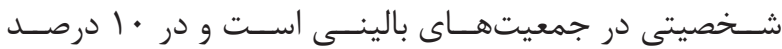

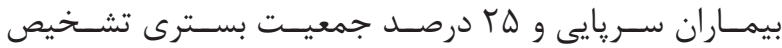

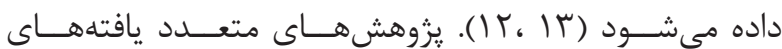

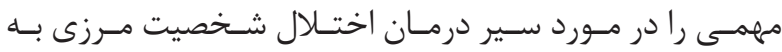

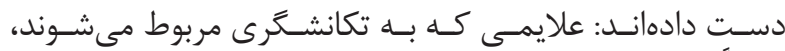

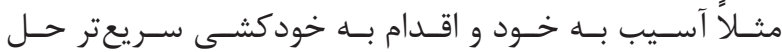

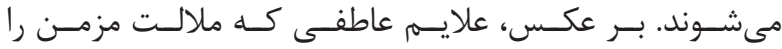

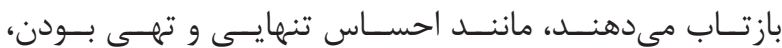

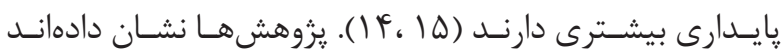

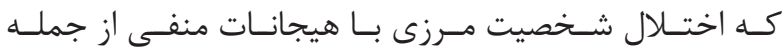

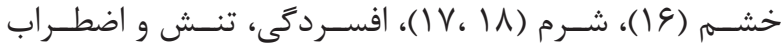

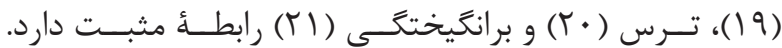

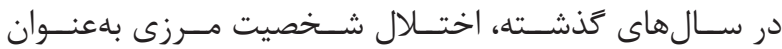

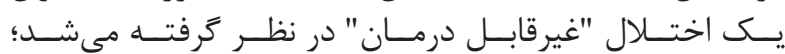

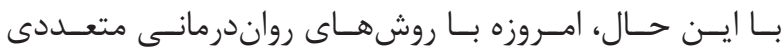

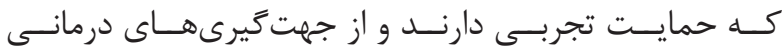

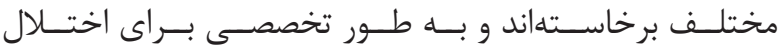

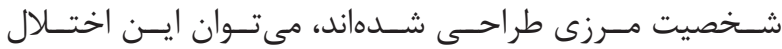

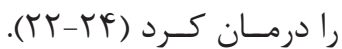

يكــى از رويكردهـــاى درمانـى مــورد اسـتفاده در اختــلال

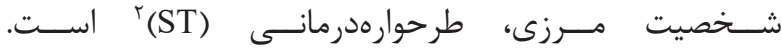

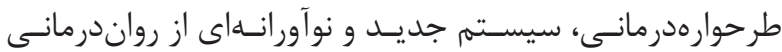

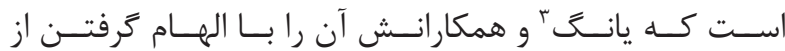

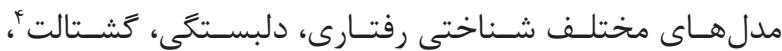

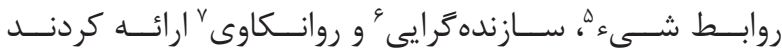

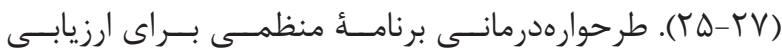

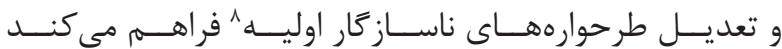

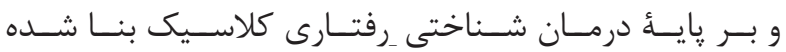

\footnotetext{
${ }^{6}$ Constructivism

${ }^{7}$ Psychoanalysis

${ }^{8}$ Early maladaptive schemas

${ }^{9}$ Giesen-Bloo

${ }^{10}$ Transference focused psychotherapy
} 


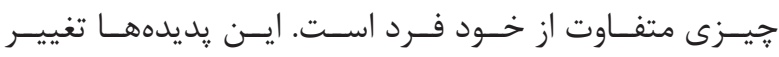

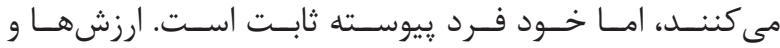

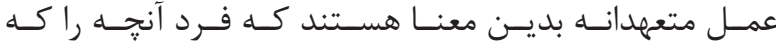

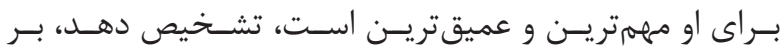

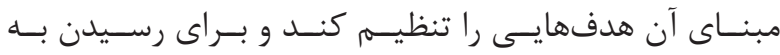

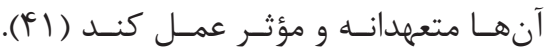

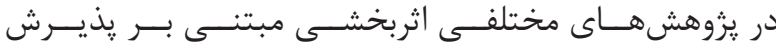

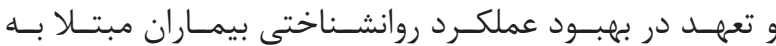

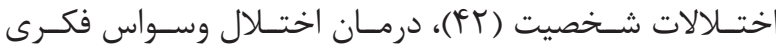

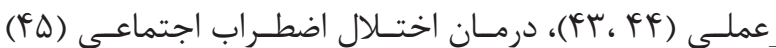
و اختـلال افسـرد

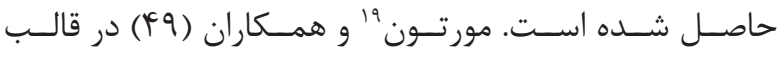

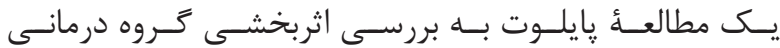

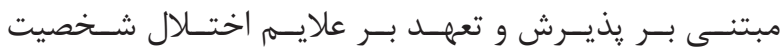

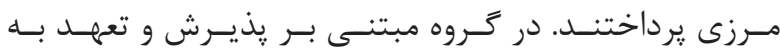

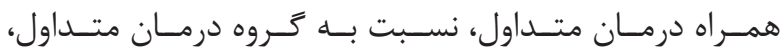

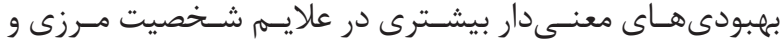

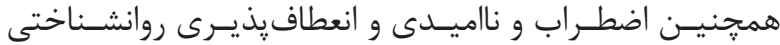

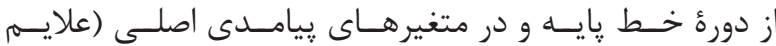

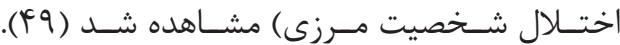

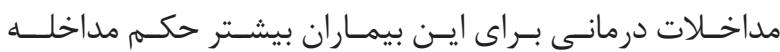

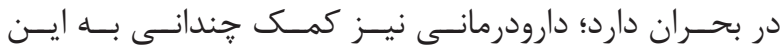

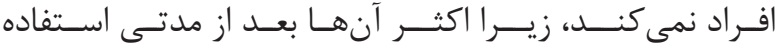

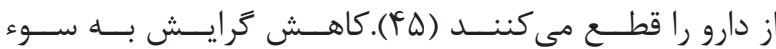

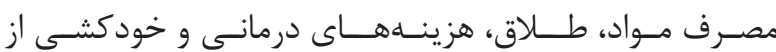

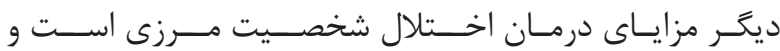

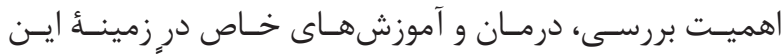

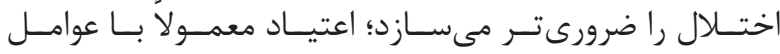

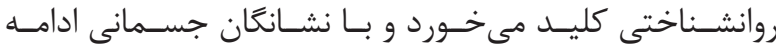

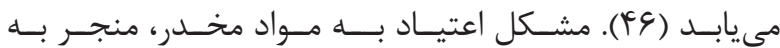

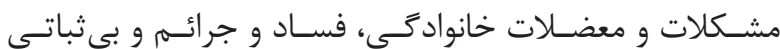

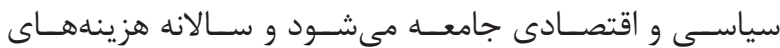

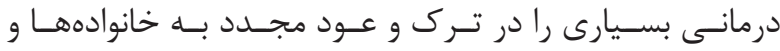

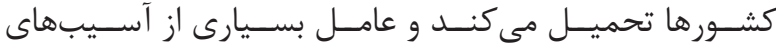

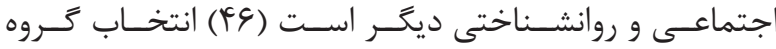

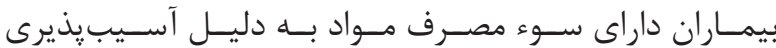

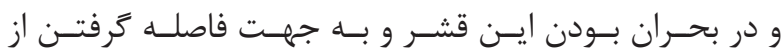

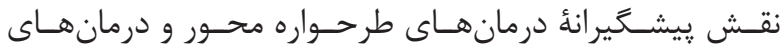

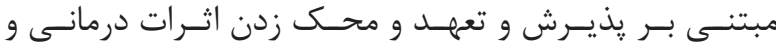

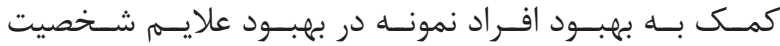

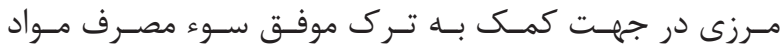

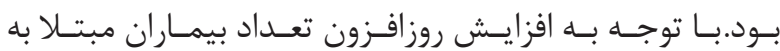

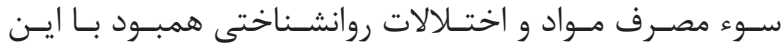

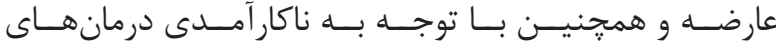

${ }^{11}$ Acceptance \& commitmen therapy

${ }^{12}$ Hayes

${ }^{13}$ Defusion

${ }^{14}$ Acceptance

${ }^{15}$ Contact with the present moment

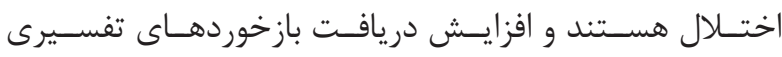

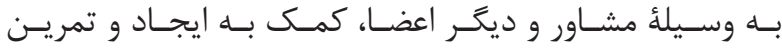

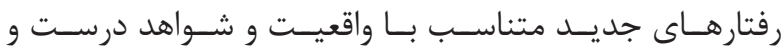

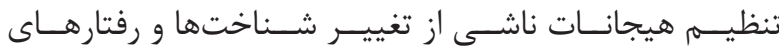

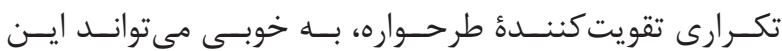

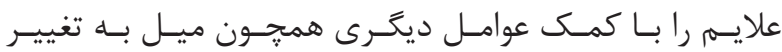

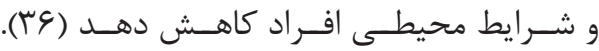

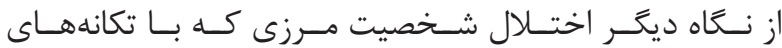

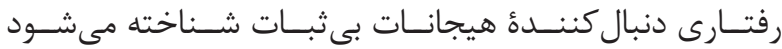

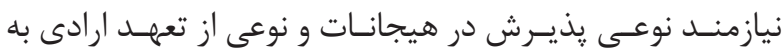

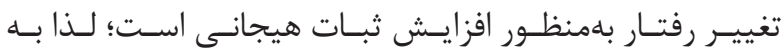

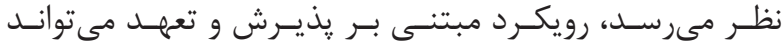

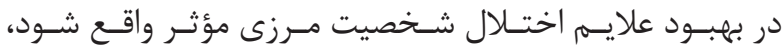

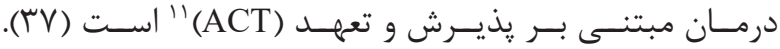

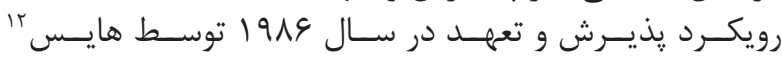

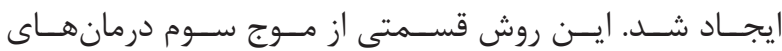

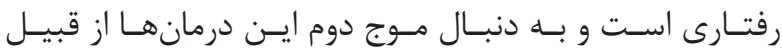

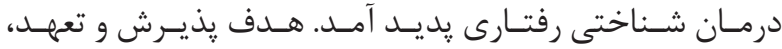

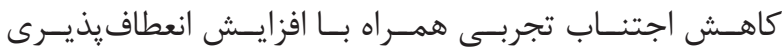

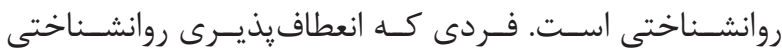

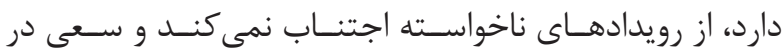

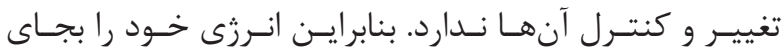

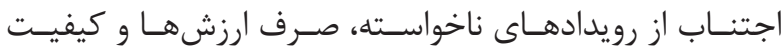

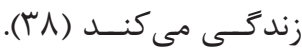

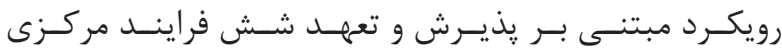

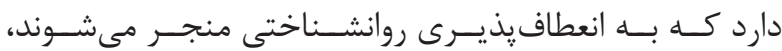

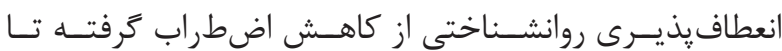

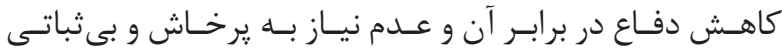

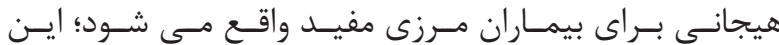

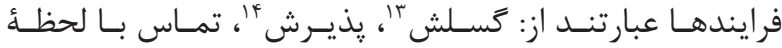

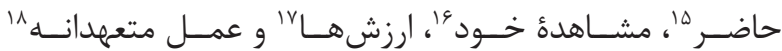

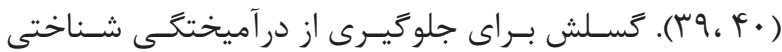

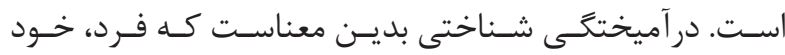

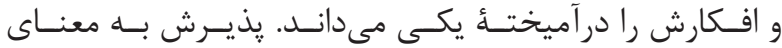

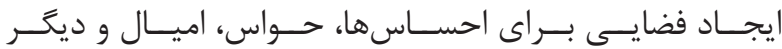

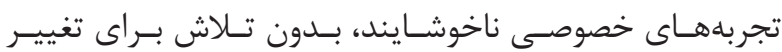

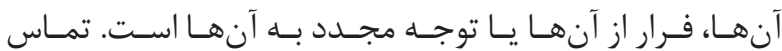

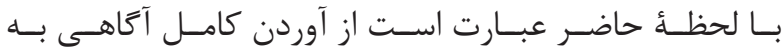

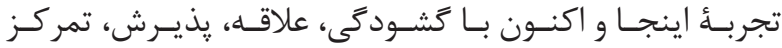

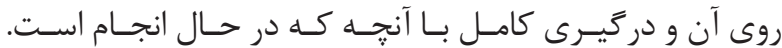

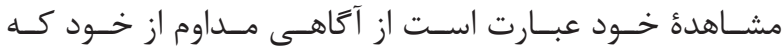

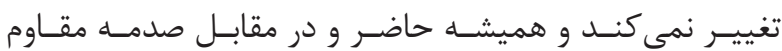

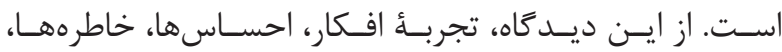

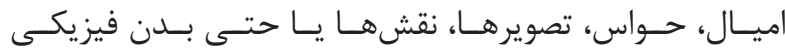

\footnotetext{
${ }^{16}$ Observing self

${ }^{17}$ Values

${ }^{18}$ Committed action

${ }^{19}$ Morton
} 


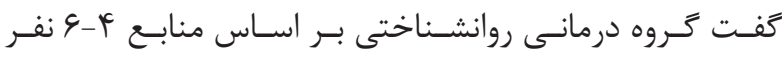

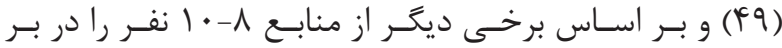

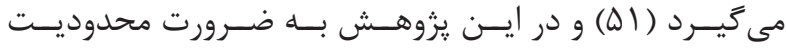

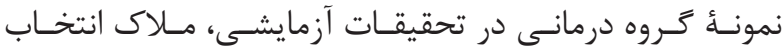

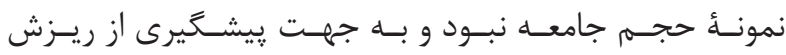

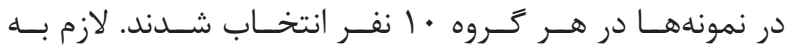

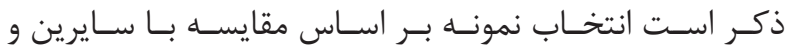

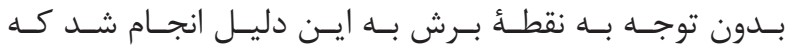

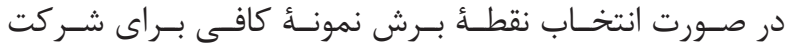

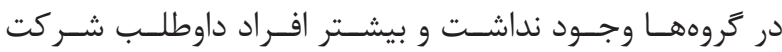

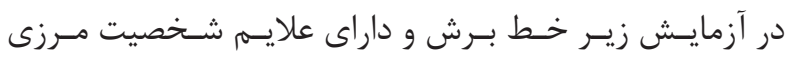

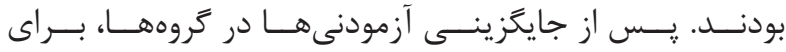

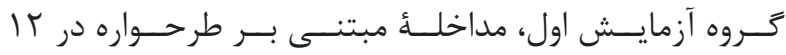

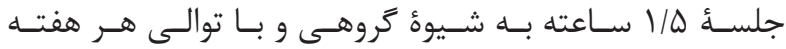

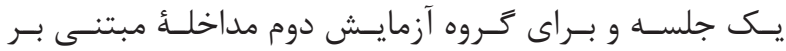

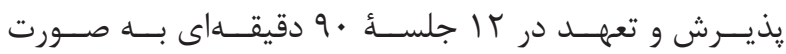

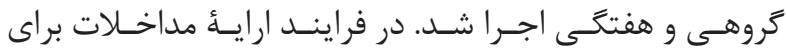

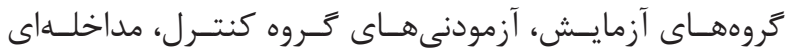

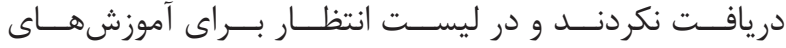

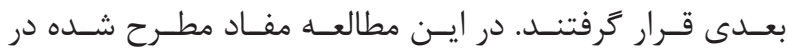

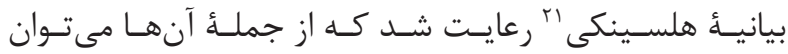

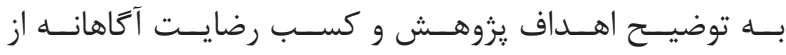

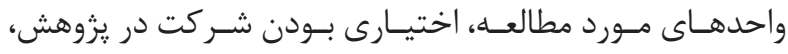

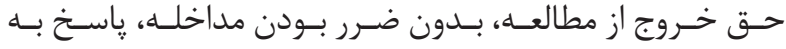

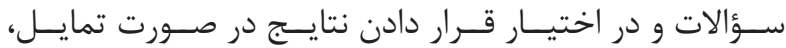

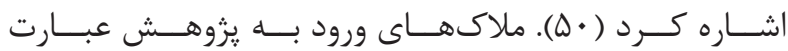

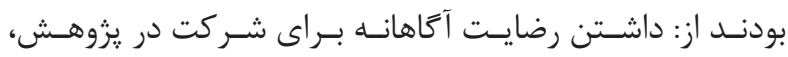

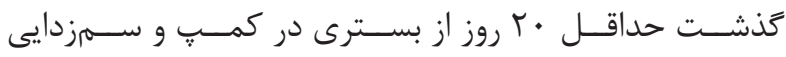

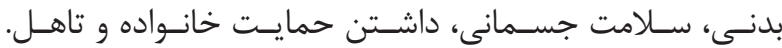

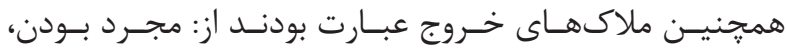

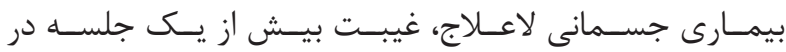

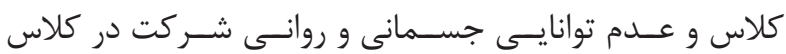

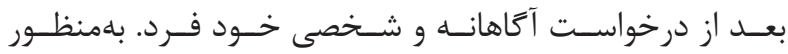

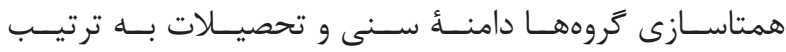

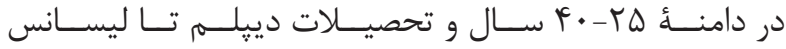

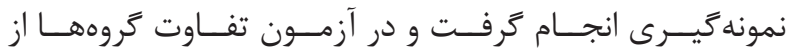

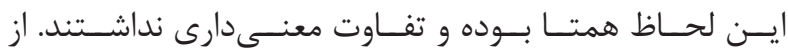

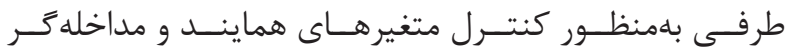

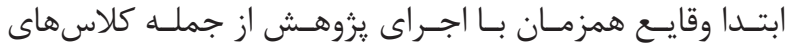

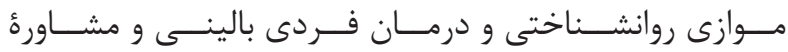

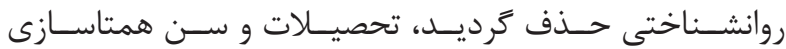

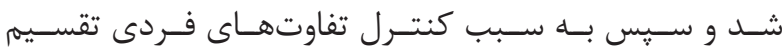

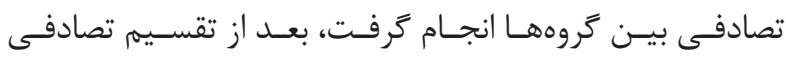

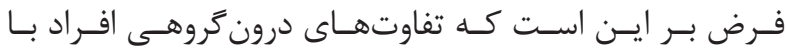

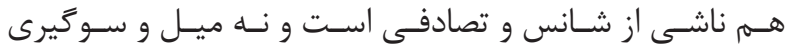

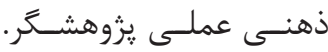

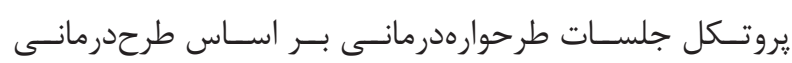

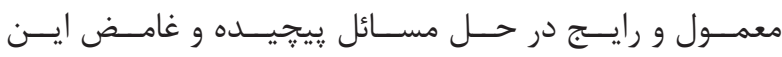

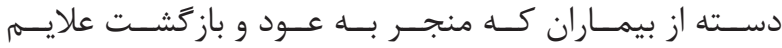

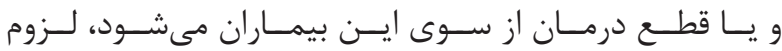

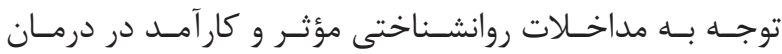

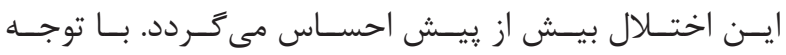

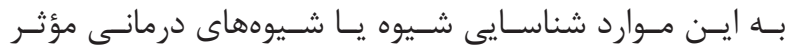

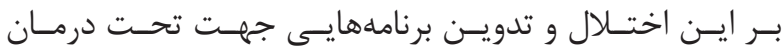

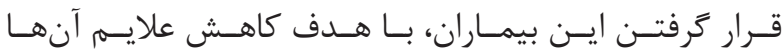

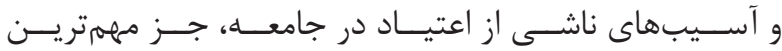

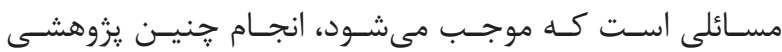

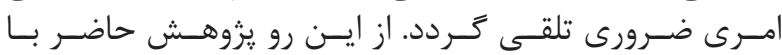

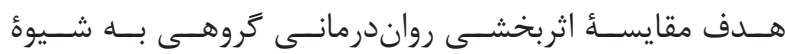

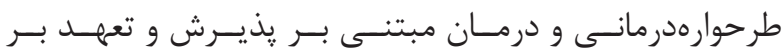

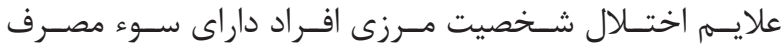

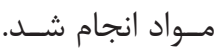

مواد و روشها

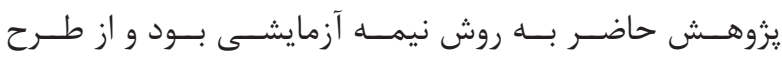

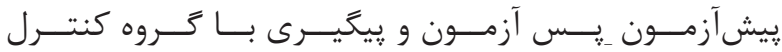

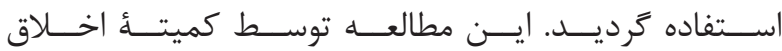

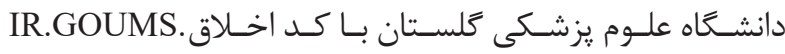

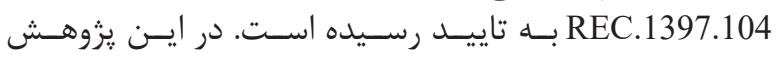

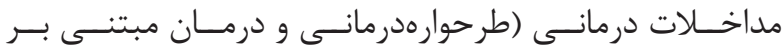

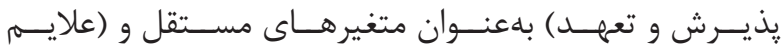

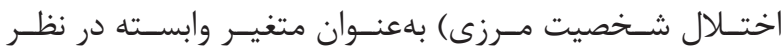

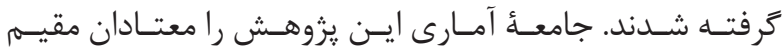

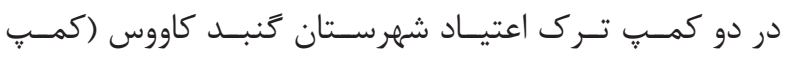

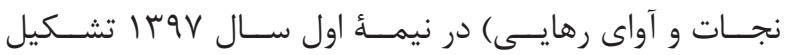

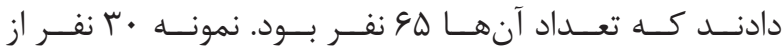

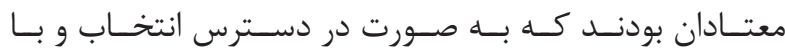

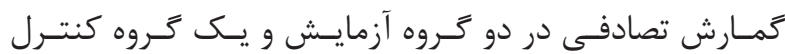

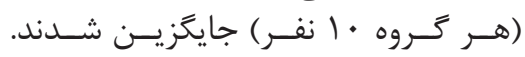

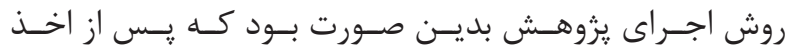

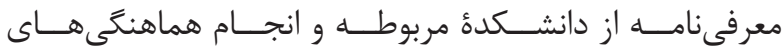

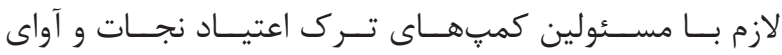

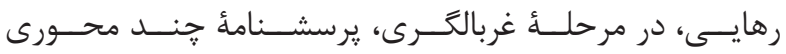

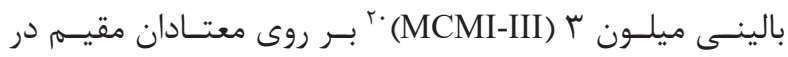

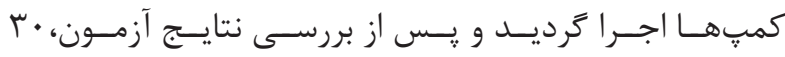

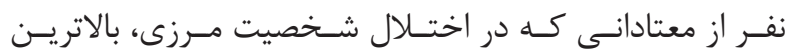

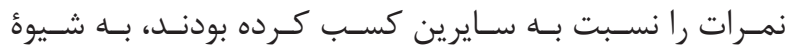

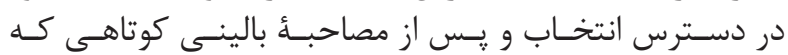

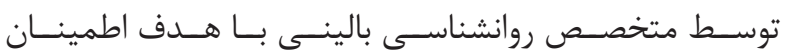

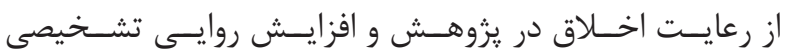

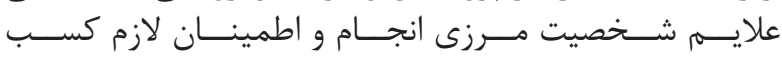

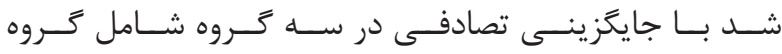

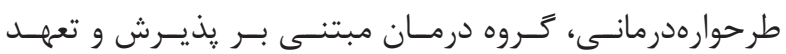

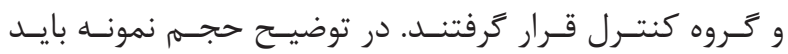




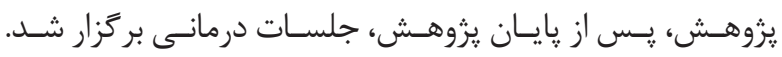
شــرح مختصــر محتــواى جلســات رواندرمانسى بـــهـ شـيوه

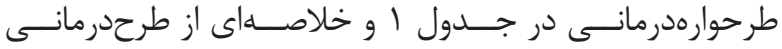

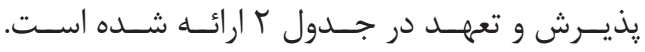

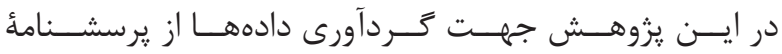

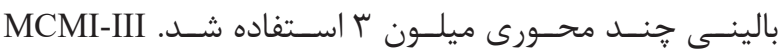

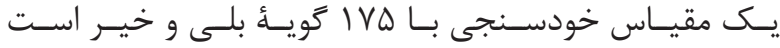

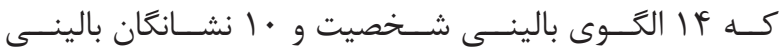

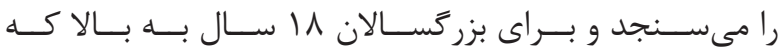

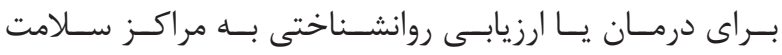

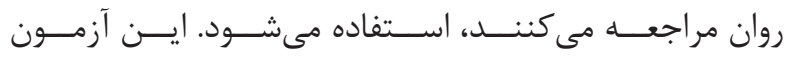

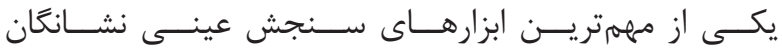

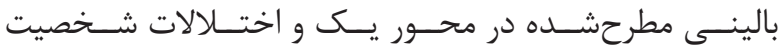

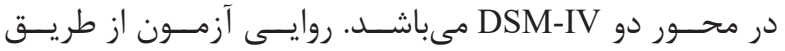

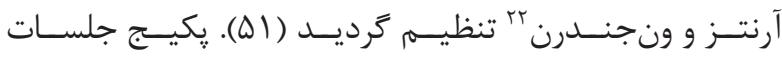

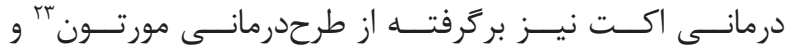

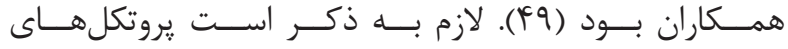

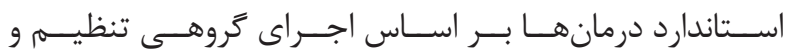

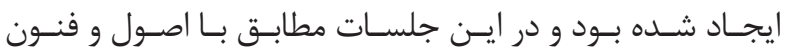

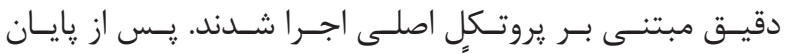

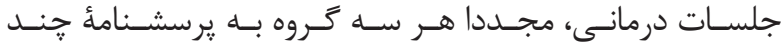

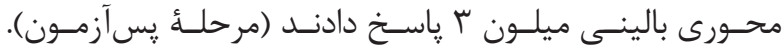

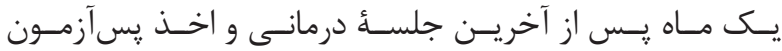

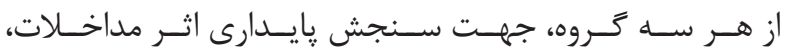

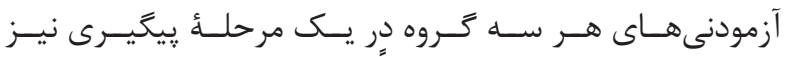

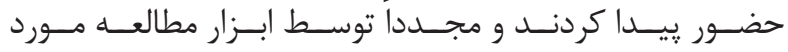

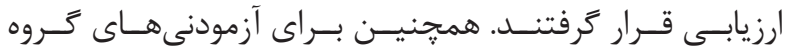

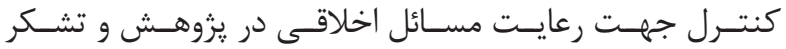

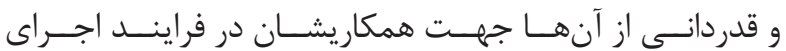

جدول ا- شرح مختصرى از جلسات رواندرمانى گروهى به شيوه طرحوارهدرمانى.

\begin{tabular}{|c|c|}
\hline 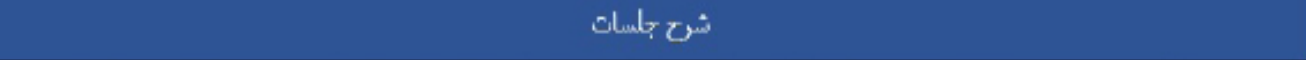 & جاستاك \\
\hline 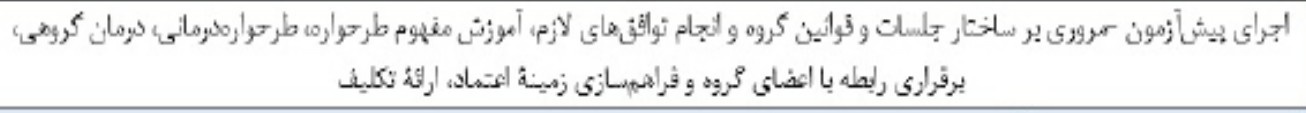 & جلسة الول \\
\hline 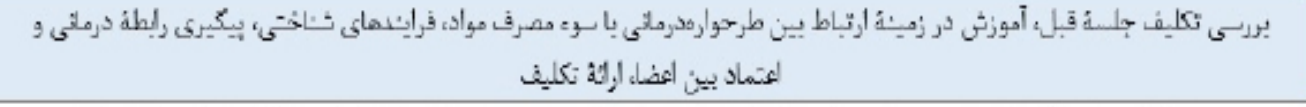 & جلسة دوم \\
\hline 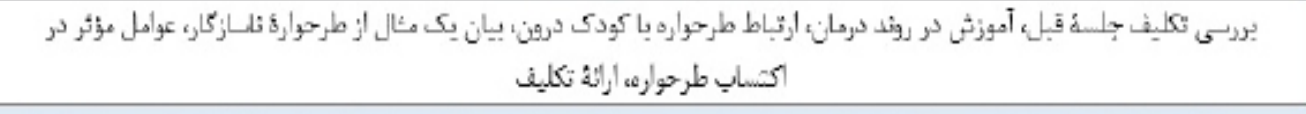 & بلسة سوم \\
\hline 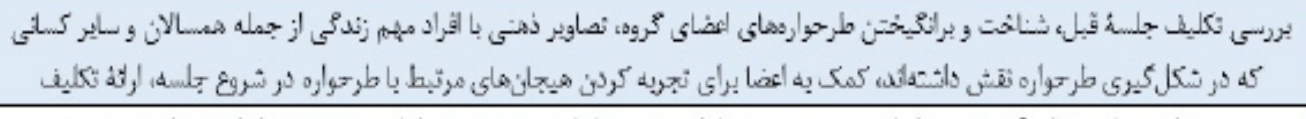 & جلسه جهارم \\
\hline 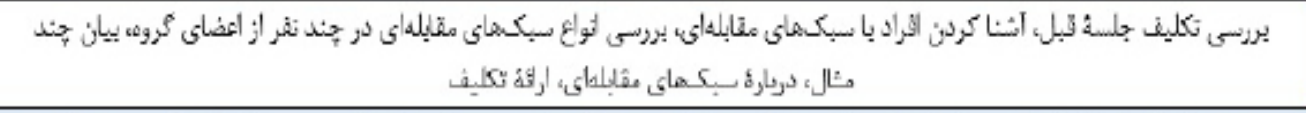 & جاسة بئجم \\
\hline 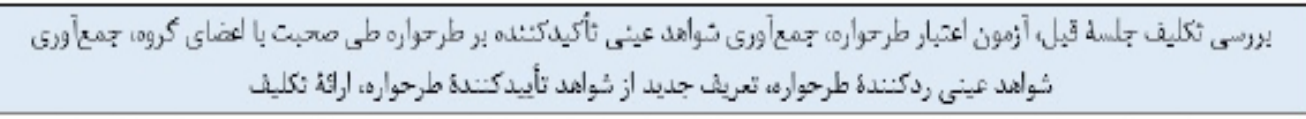 & جلسة ثـثم \\
\hline 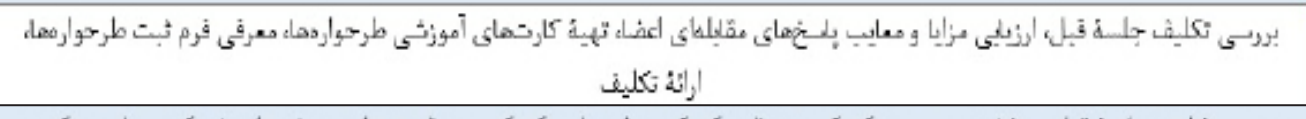 & جلسة هلقتم \\
\hline 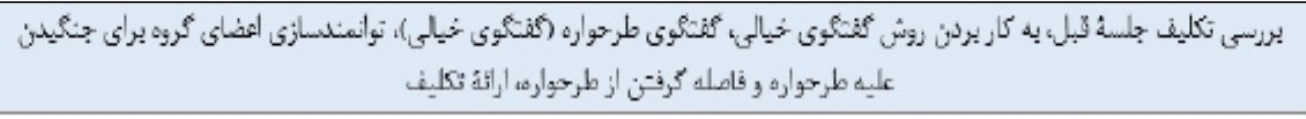 & T \\
\hline 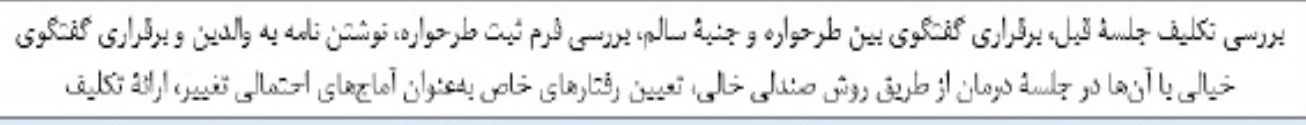 & جلسة تهم \\
\hline 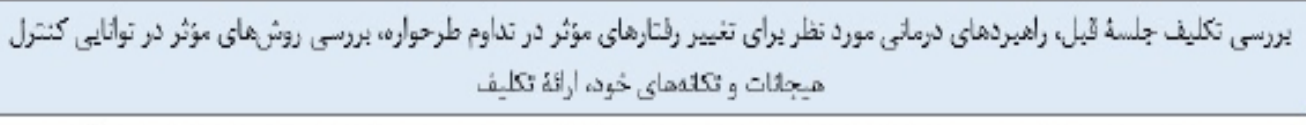 & 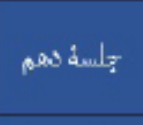 \\
\hline 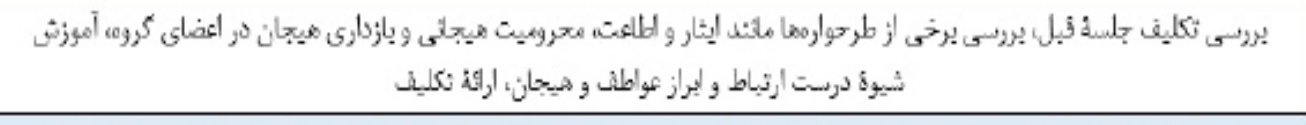 & جلسة يازيمهم \\
\hline 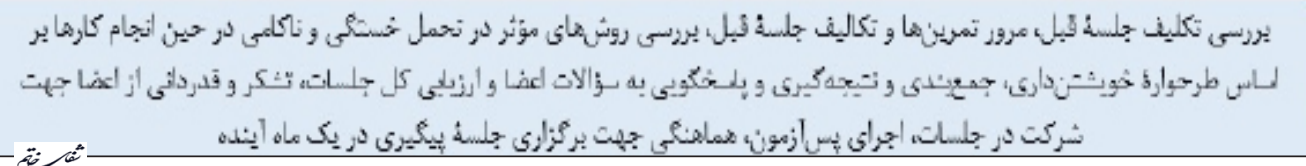 & جلسةُ دوازدصم \\
\hline
\end{tabular}


جدول r- مختصرى از محتواى جلسات رواندرمانى كروهى به شيوة پِيرش و تعهد.

\section{شع بلسات}

بلسات

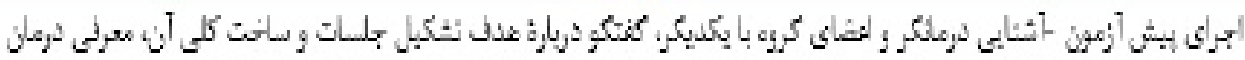

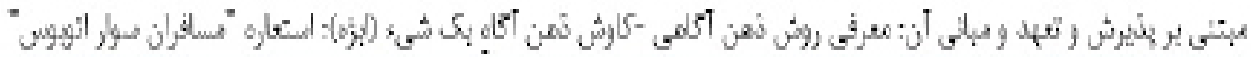

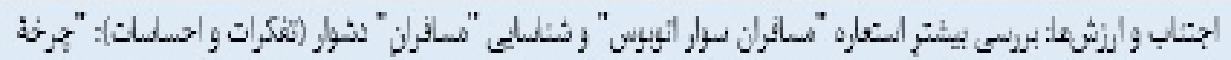

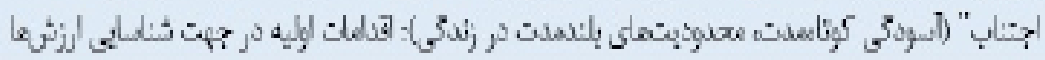

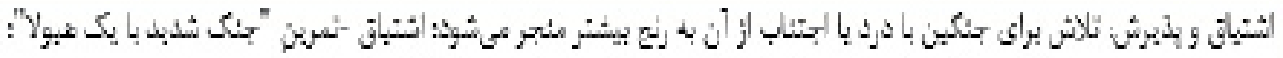

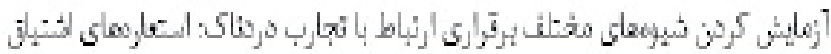

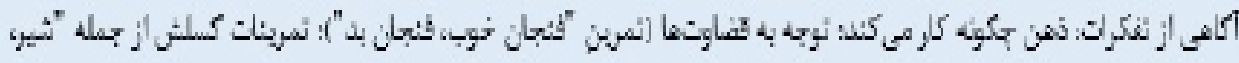

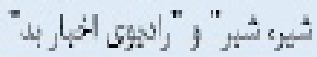

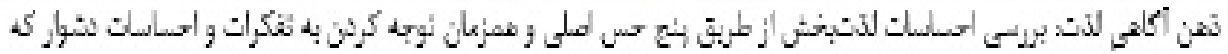

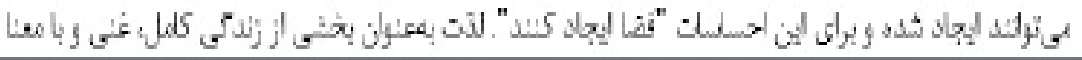

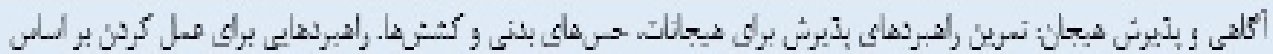

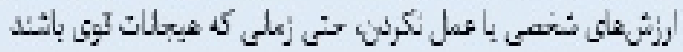

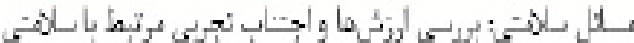

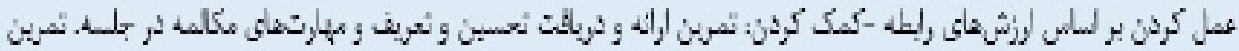

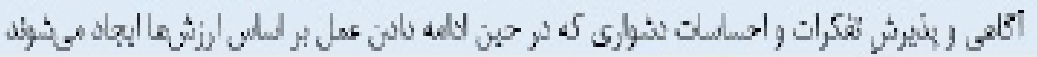

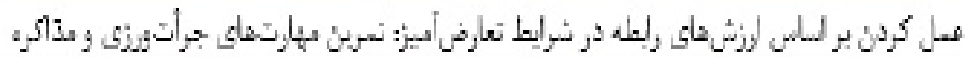

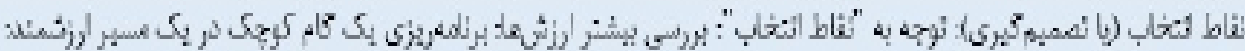

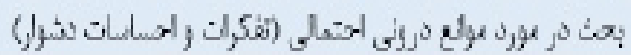

\begin{tabular}{|c|c|}
\hline 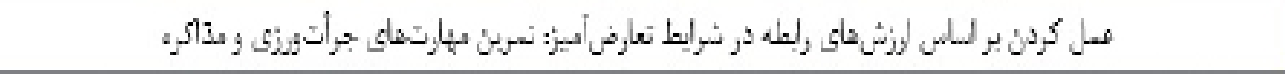 & بلس نهم \\
\hline 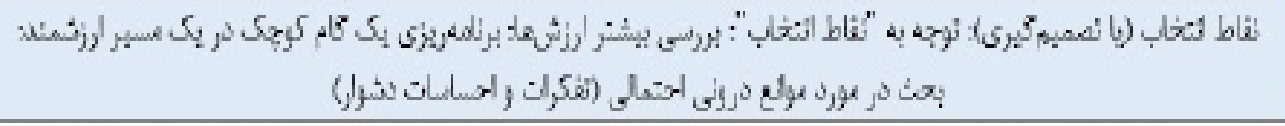 & بلس لغيم \\
\hline 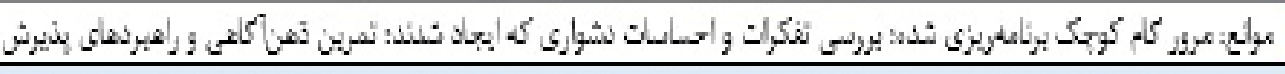 & جلسن يازيعم \\
\hline 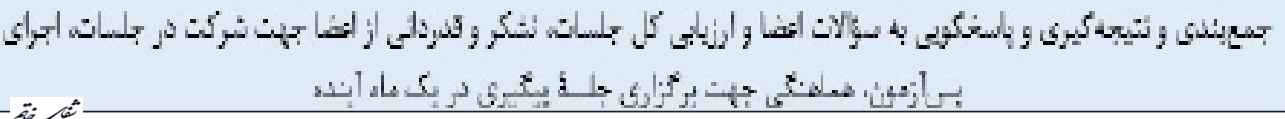 & جلسل ثوازئمه \\
\hline
\end{tabular}

SPSS

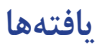

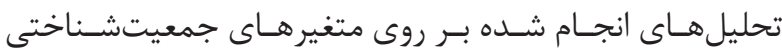

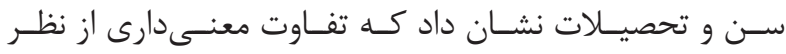
سـن (F)

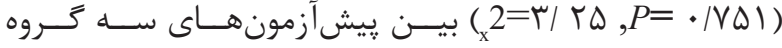

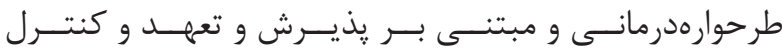

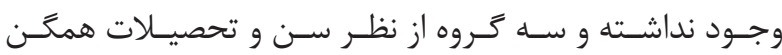

هسبـتند.

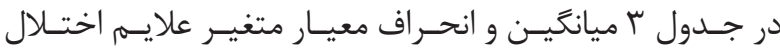

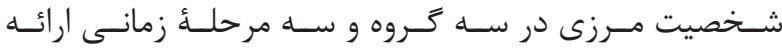

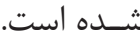

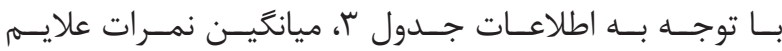

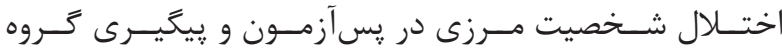

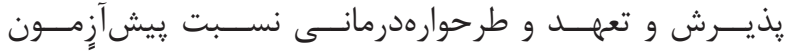

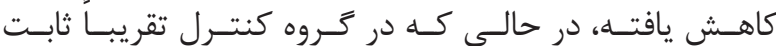

مانده اسـت كانت

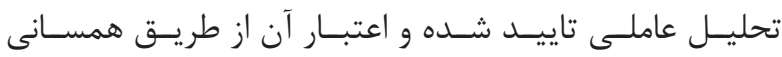

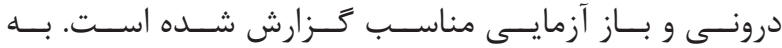

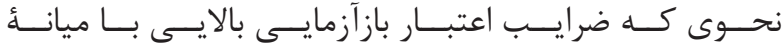

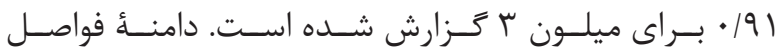

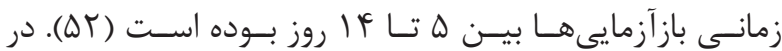

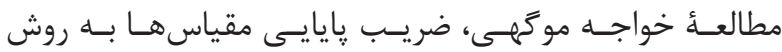

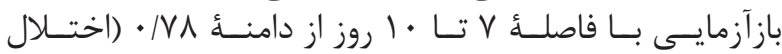

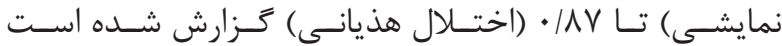

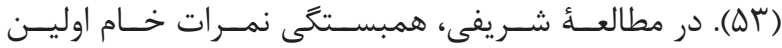

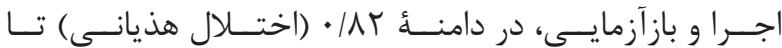

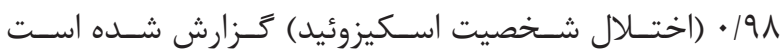

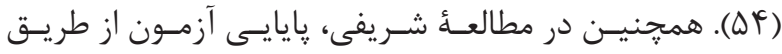

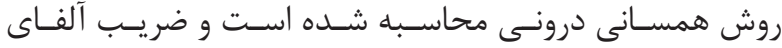

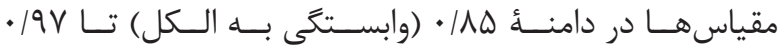

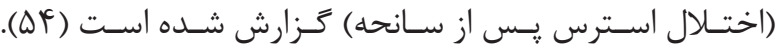

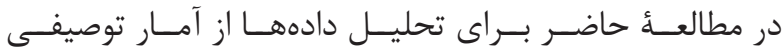

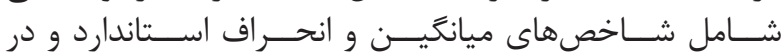

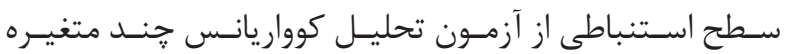

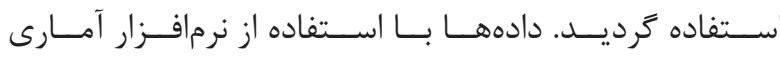




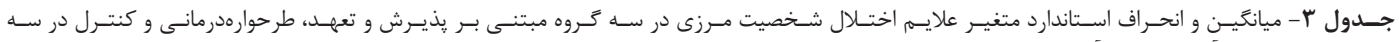

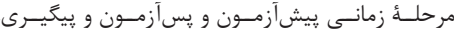

\begin{tabular}{|c|c|c|c|c|c|c|c|}
\hline \multicolumn{2}{|c|}{ بِئَيرى } & \multicolumn{2}{|c|}{ بِسَآزمون } & \multicolumn{2}{|c|}{ بيشى آزمون } & \multirow{2}{*}{\multicolumn{2}{|c|}{ متغروه }} \\
\hline ميائكين & انحراف استائدارد & ميائينين & ميانكّين & انحراق استاندارد & ميائّين & & \\
\hline$V / \Delta \Delta$ & $\mathrm{Fq} / \mathrm{A}$. & $9 / 49$ & $F N / G$. & $N \mid 9 V$ & $\Delta \varphi$ & 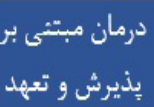 & \\
\hline$q / 4 r$ & $f y / 1$. & $9 / 4 f$ & $f \mid / r$. & $11 / \cdot v$ & $\Delta \cdot / \Delta \cdot$ & طرحوار هدرمانى & (5) \\
\hline $9 / 97$ & $\Delta N / 11$ & $1 \cdot / \% V$ & $\Delta V / T$. & 1.111 & $\Delta \Lambda$ & كثترل & \\
\hline
\end{tabular}

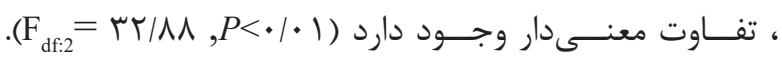

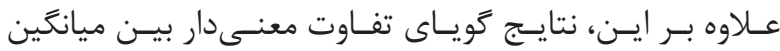

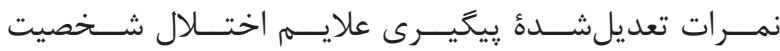

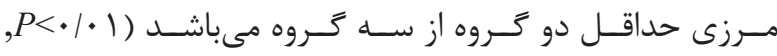
. $\left(\mathrm{F}_{\mathrm{df}: 2}=\mathrm{rr} / \mathrm{V}\right.$.

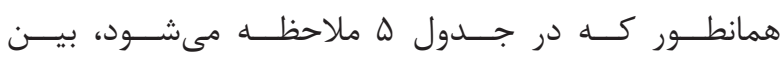

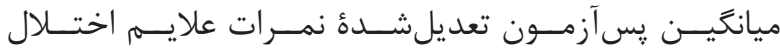

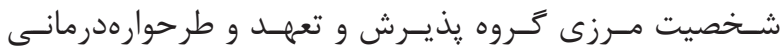

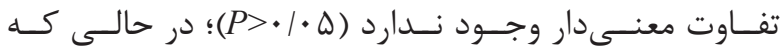

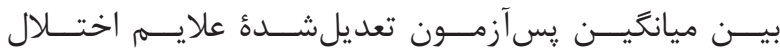

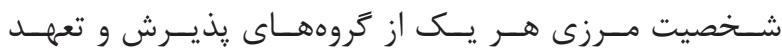

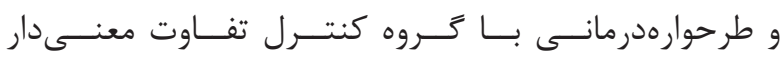

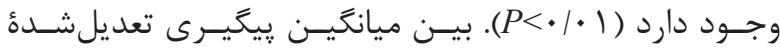

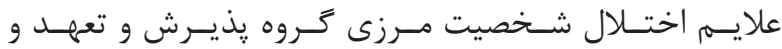

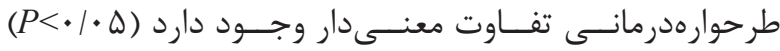

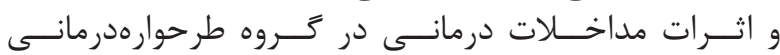

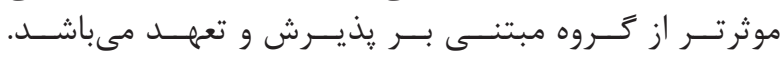

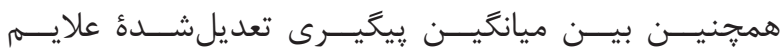

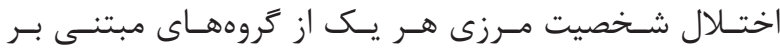

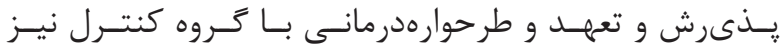

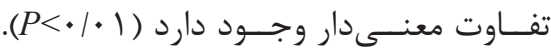

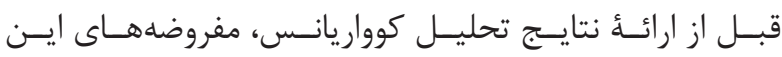

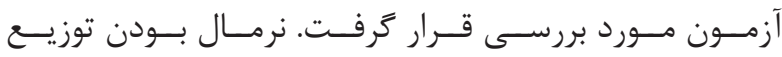

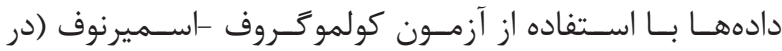

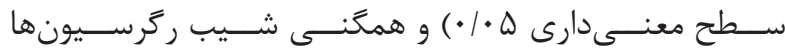

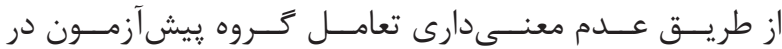

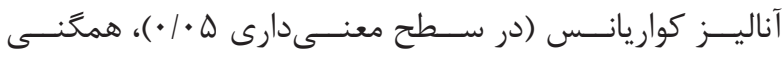

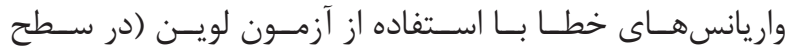

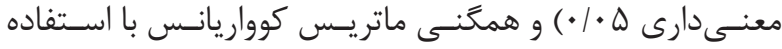

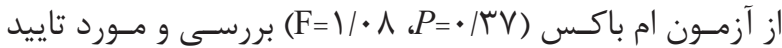

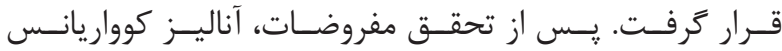

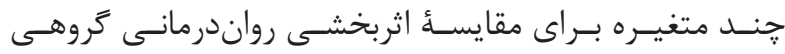

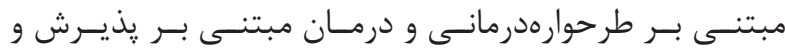

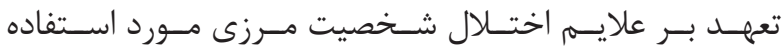

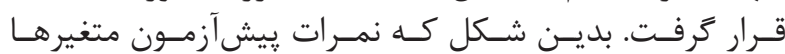

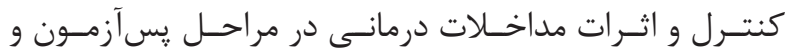

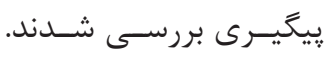

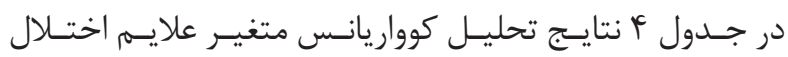

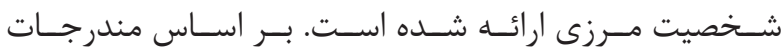

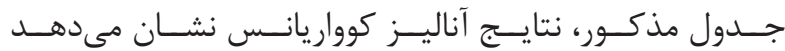

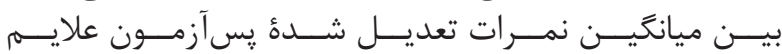

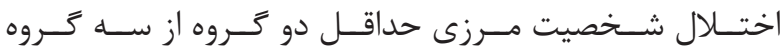

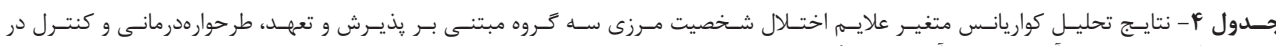

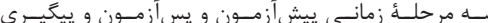

\begin{tabular}{|c|c|c|c|c|c|c|c|c|}
\hline توان آزمون & مجذور اتا & معثى دارى & F & ميائكين مجذورات & درجهُ آزادى & مجموع مجذورات & مرحله & متغير وابسته \\
\hline 1 & . $/$ VIV & $+1 \cdots$ & MY/AN & YHY/fY & $r$ & $f \notin F / A V$ & بِس آزمون & عاليم اختلال \\
\hline 1 & - IVYY & $.1 \cdots$ & $r r / v$. & rG./VD & r & $\Delta Y Y / \Delta I$ & بِيكّيرى & شخصِت مرزى \\
\hline
\end{tabular}

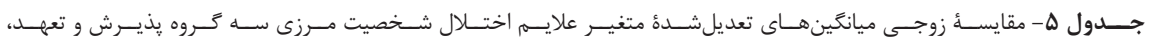

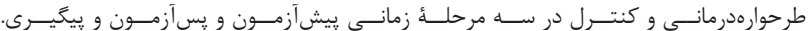

\begin{tabular}{|c|c|c|c|c|c|}
\hline \multicolumn{5}{|c|}{ مياعين } & \multirow{2}{*}{ متغير وايسته } \\
\hline معنى دارى & خطاى الستالدارد & تقاوت مياتكينها & مقايسه & مبدا & \\
\hline$\cdot 11 \cdot 4$ & $1 / 4 T$ & r/NT & طرحوارهدرمانى & \multirow{2}{*}{ بذيرش و نعهيد } & \multirow{4}{*}{ بسآزمون علايم اختالال شخصيت مرزى } \\
\hline$\cdot / \cdots$ & $1 / 19$ & $9 / 99$ 룽 & كنترل & & \\
\hline$\cdot 1 \cdot f$ & $1 / K T$ & $-r / N$ & بذيرش و تعهد & \multirow{2}{*}{ طرحوارمدرمانى } & \\
\hline$\cdot / \cdots$ & $1 / r 4$ & $9 / Y Y *$ & كتئرل & & \\
\hline $.1 .1 f$ & $1 / r V$ & $r / 9 V *$ & طرحوارمدرمانى & \multirow{2}{*}{ يُديرش و تعهيد } & \multirow{4}{*}{ بيّيرى علايه اختلال شخصيت مرزى } \\
\hline$\cdot 1 \cdots$ & $1 / F^{*}$ & $-9 / 094$ & كنترل & & \\
\hline .1 .14 & $1 / r V$ & $r / 9 v *$ & يذيرش و تعهد & \multirow{2}{*}{ طرحواردنرمانى } & \\
\hline $.1 \ldots$ & $1 / r$. & $-1 \cdot 109$ 粗 & كنترل & & \\
\hline
\end{tabular}




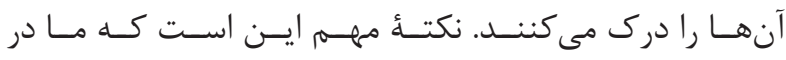

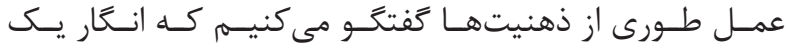

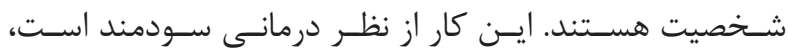

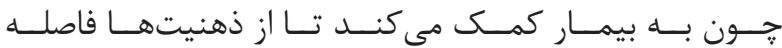

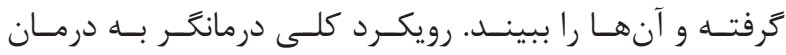

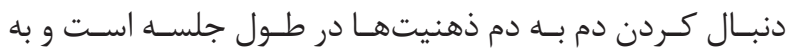

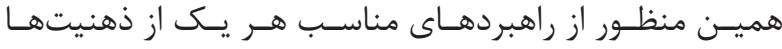

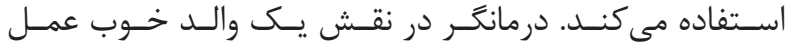

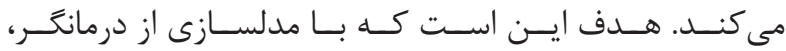

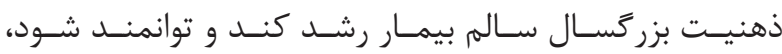

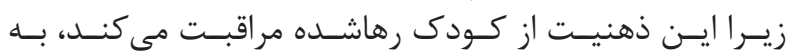

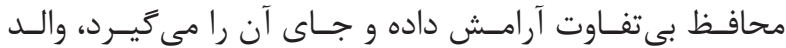

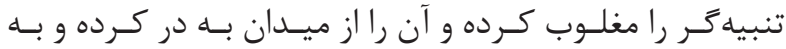

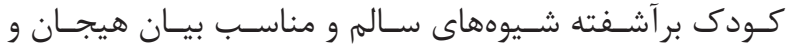

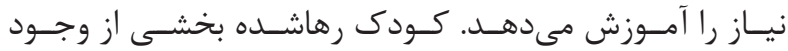

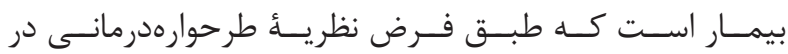

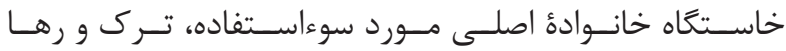

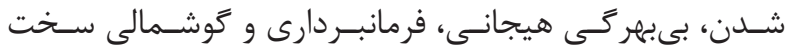

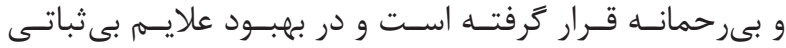

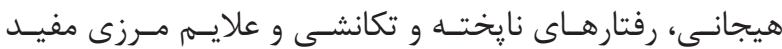

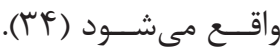

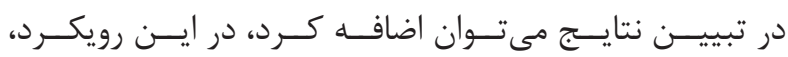

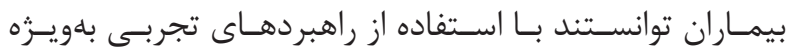

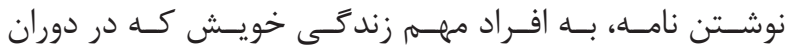

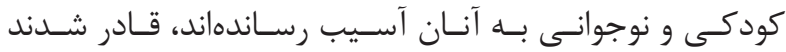

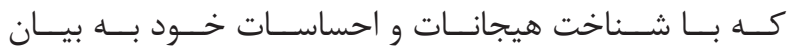

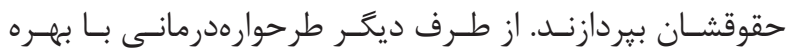

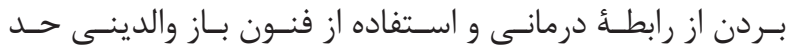

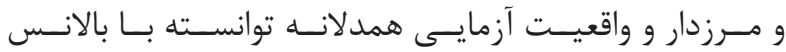

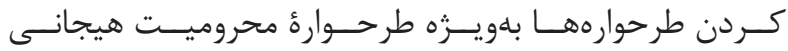

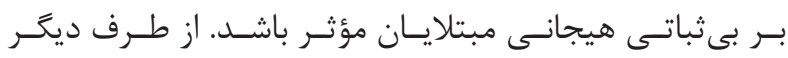

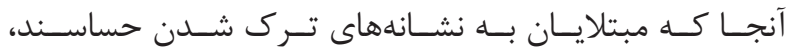

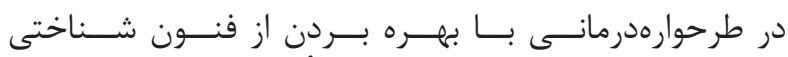

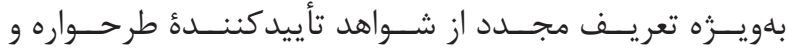

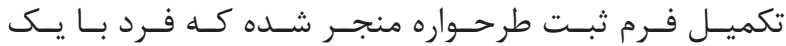

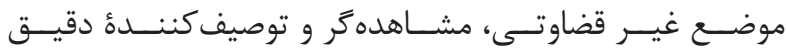

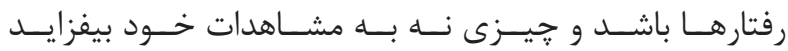

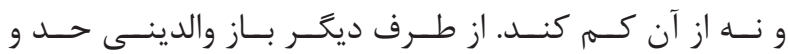

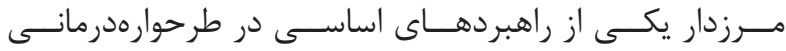

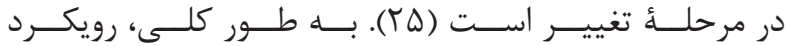

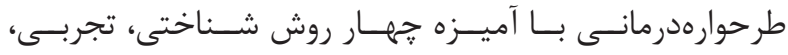

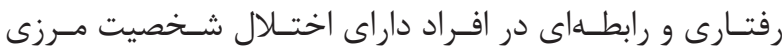

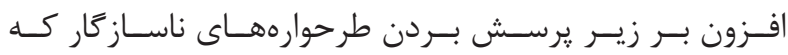

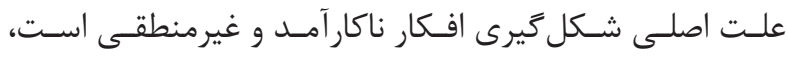

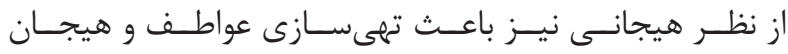

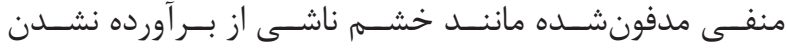

${ }^{24}$ Tan

${ }^{25} \mathrm{Jacob}$

${ }^{26}$ Nadort
بحث و نتيجه

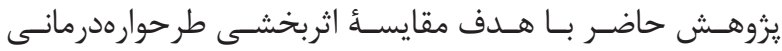

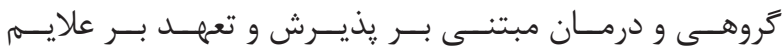

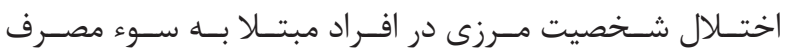

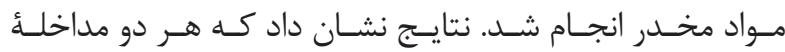

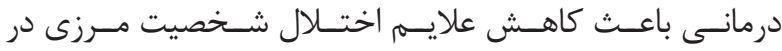

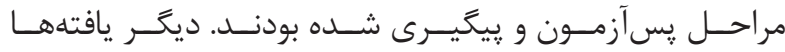

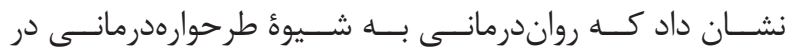

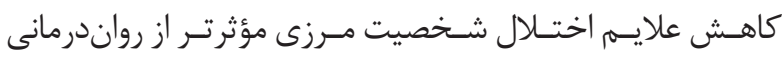

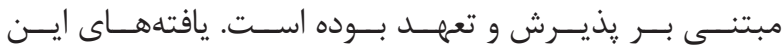

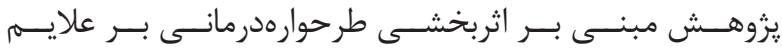

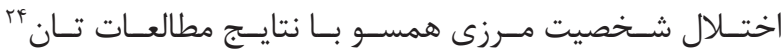

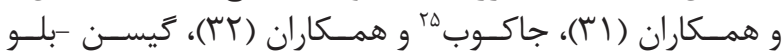

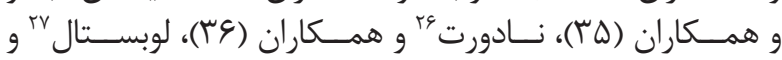

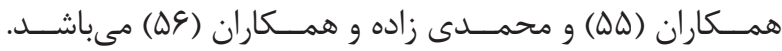

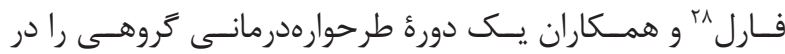

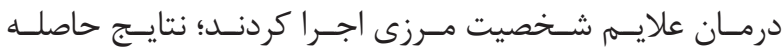

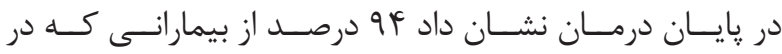

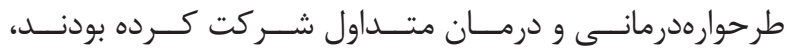

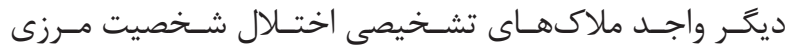

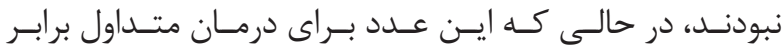

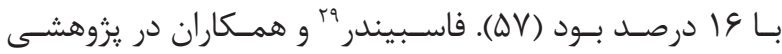

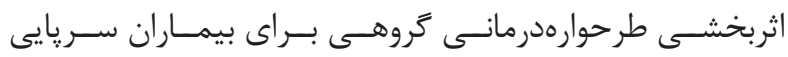

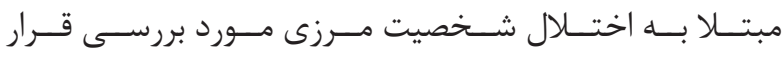

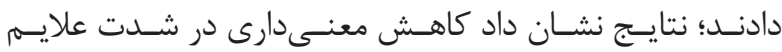

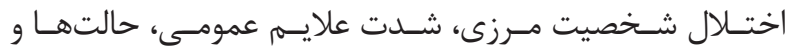

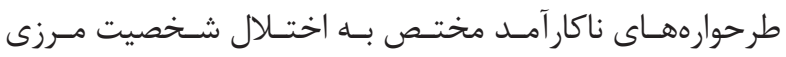

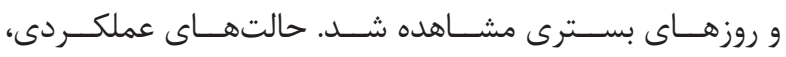

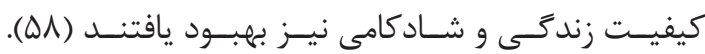
در تبييـن اثربخشـى طرحوارهدرمانسى در بهبـــود علايسـمه

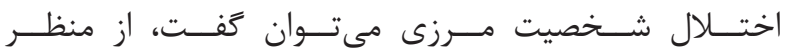

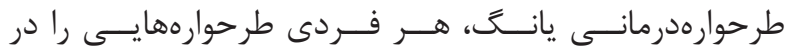

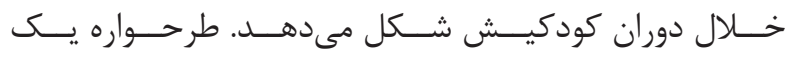

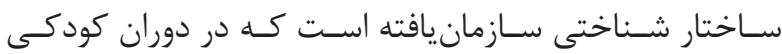

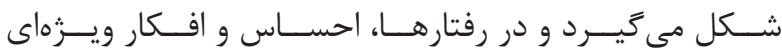

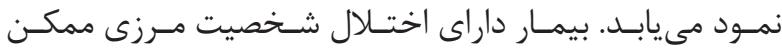

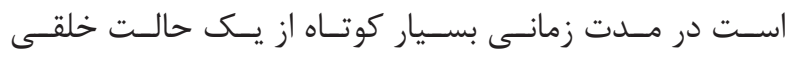

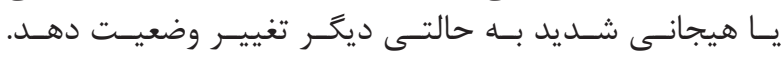

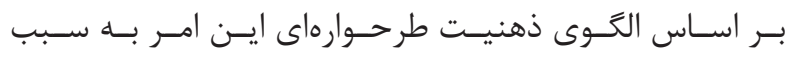

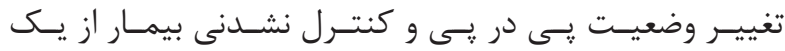

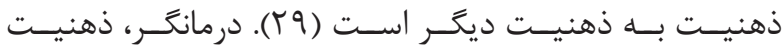

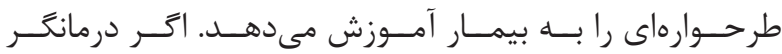

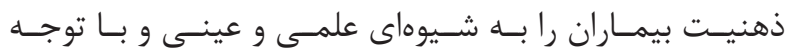

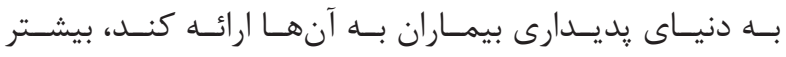

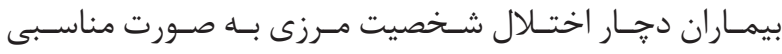

\footnotetext{
${ }^{27}$ Lobbestael

${ }^{28}$ Farrell

${ }^{29}$ Fassbinder
} 


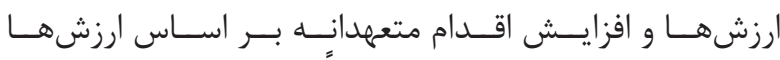

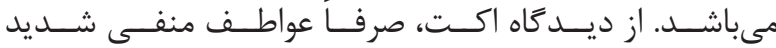

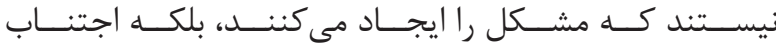

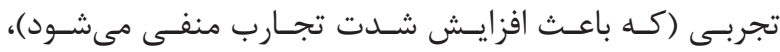

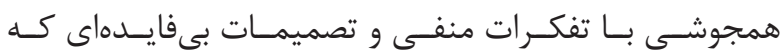

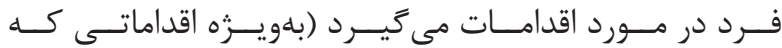

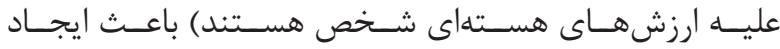

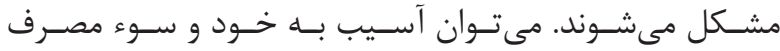

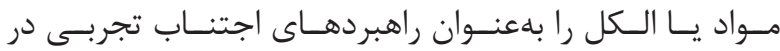

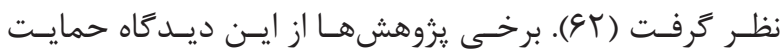

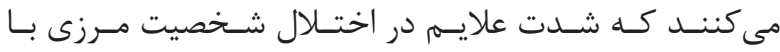

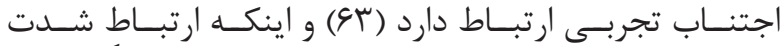

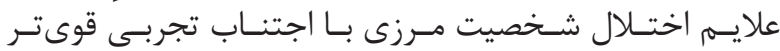

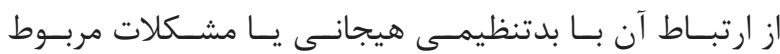

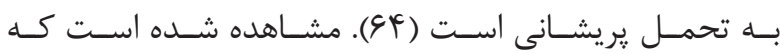

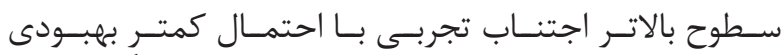

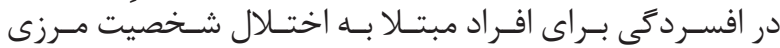

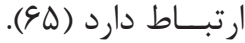

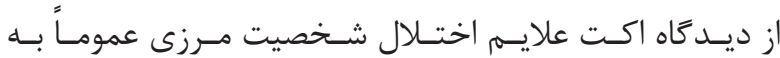

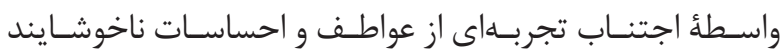

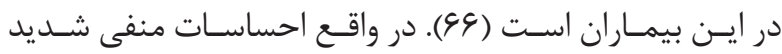

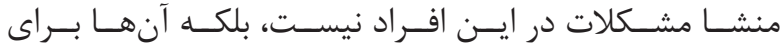

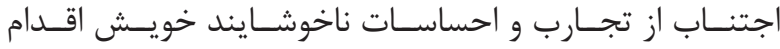

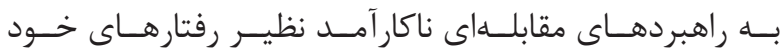

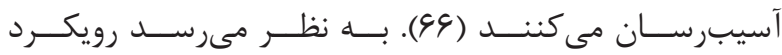

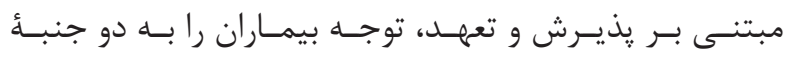

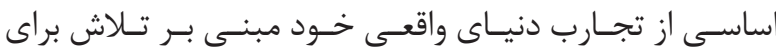

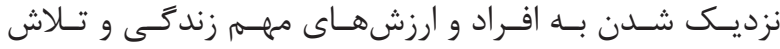

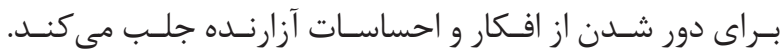

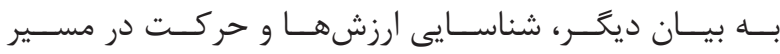

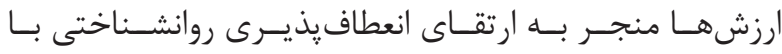

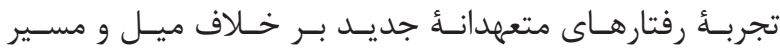

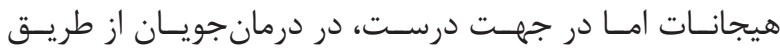

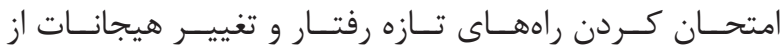

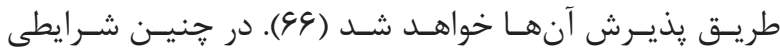

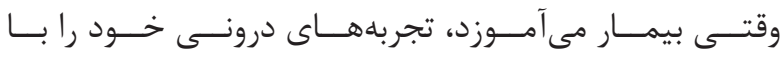

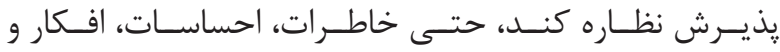

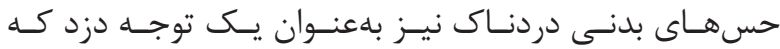

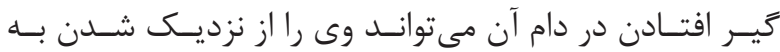

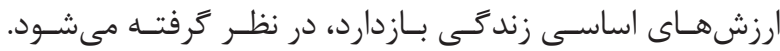

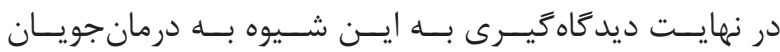

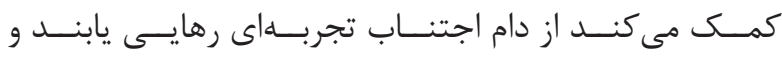

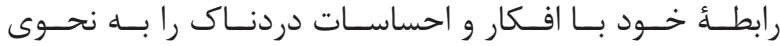

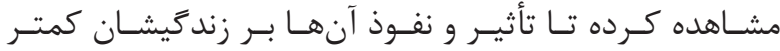

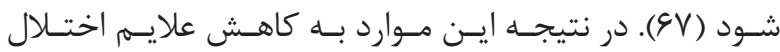

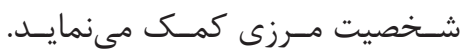

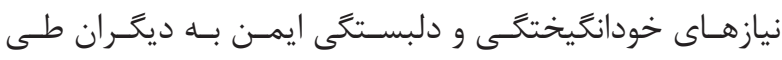

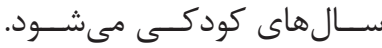

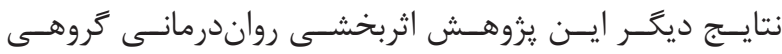

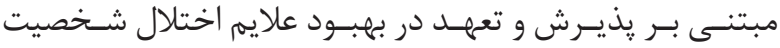

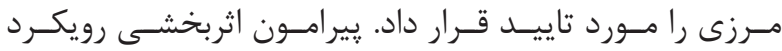

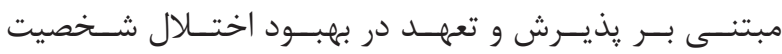

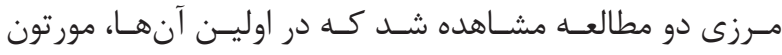

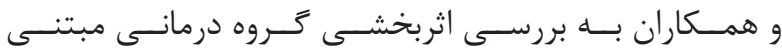

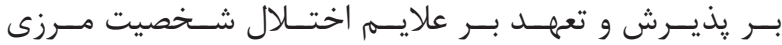

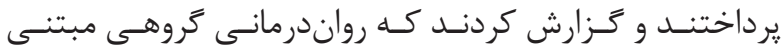

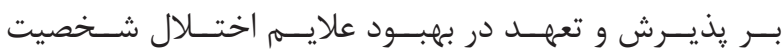

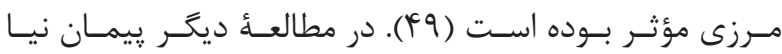

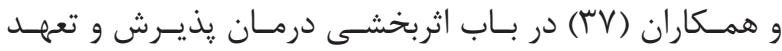

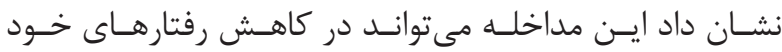

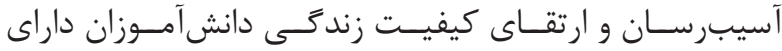

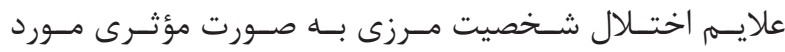

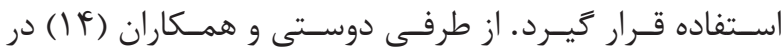

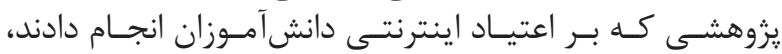

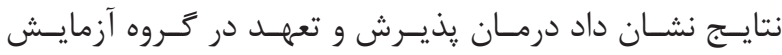

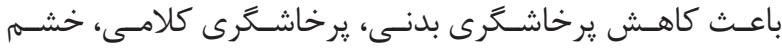

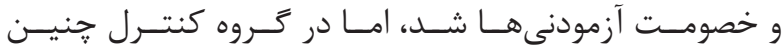

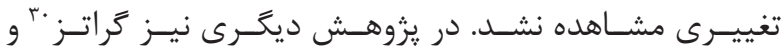

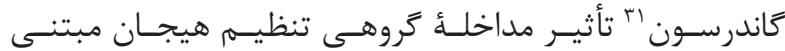

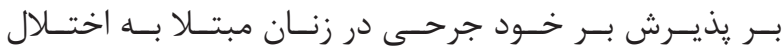

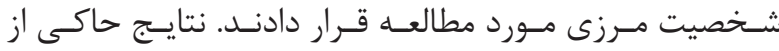

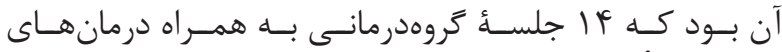

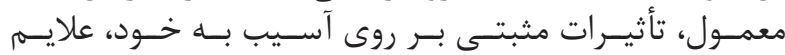

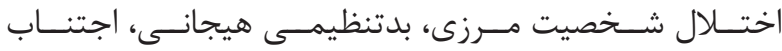

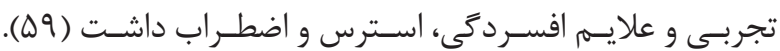

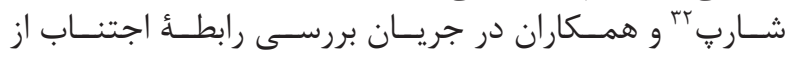

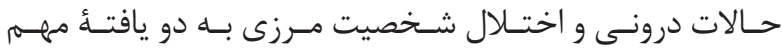

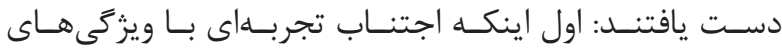

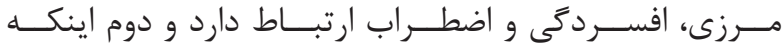

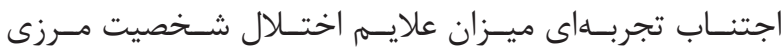

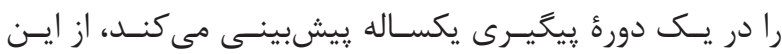

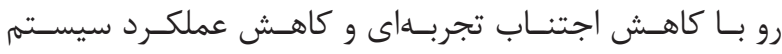

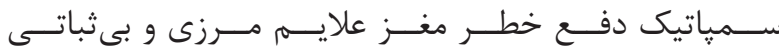

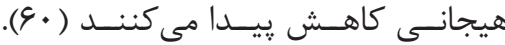

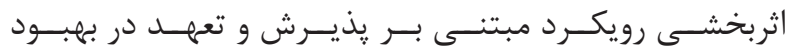

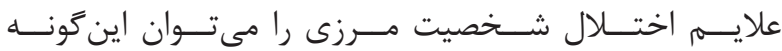

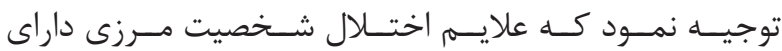

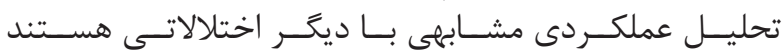

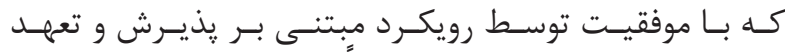

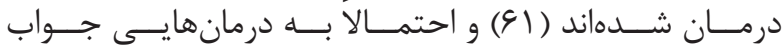

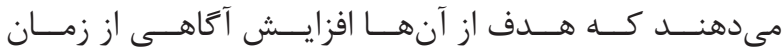

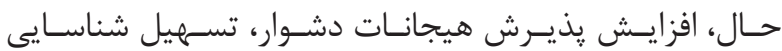

${ }^{30}$ Gratz

${ }^{31}$ Gunderson

${ }^{32}$ Sharp 


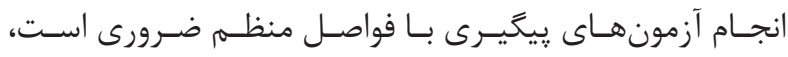

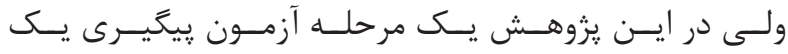

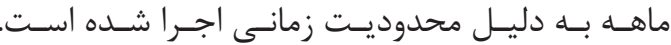

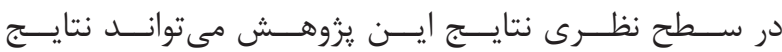

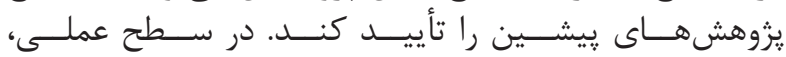

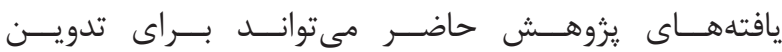

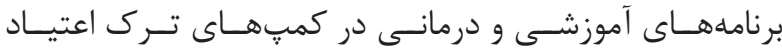

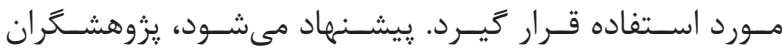

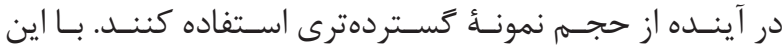

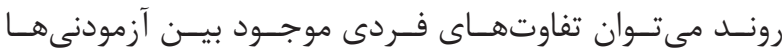

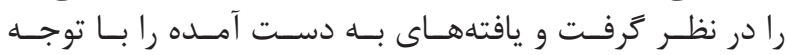

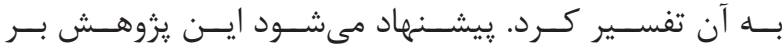

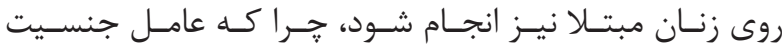

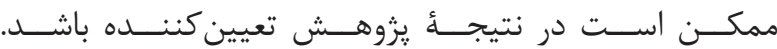

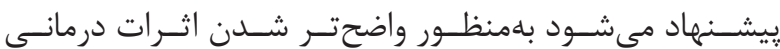

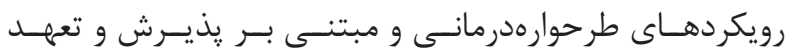

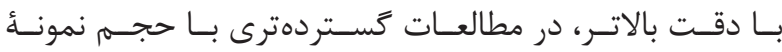

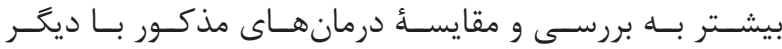

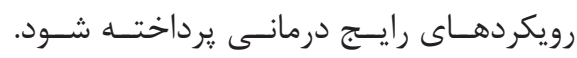

$$
\text { تشكر و قدردانى }
$$

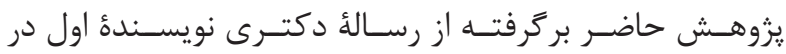

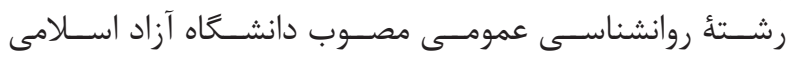

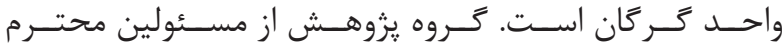

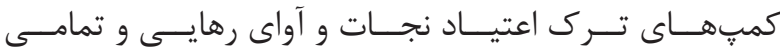

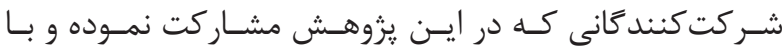

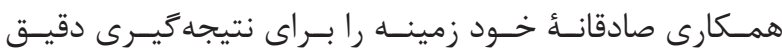

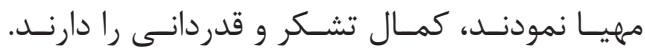

1. American Psychiatric Association. Diagnostic and statistical manual of mental disorders (DSM-IV). $5^{\text {th }}$ ed. Washington: American Psychiatric Press; 2013.

2. Tomko RL, Trull TJ, Wood PK, Sher KJ. Characteris tics of borderline personality disorder in a community sample: comorbidity, treatment utilization, and general functioning. J Pers Disord. 2014; 28(5): 734-50.

3. IsHak WW, Elbau I, Ismail A, Delaloye S, Ha K, Bolotaulo NI, et al. Quality of life in borderline personality disorder. Harv Rev Psychiatry. 2013; 21(3): 138-50.

4. Ansell EB, Sanislow CA, McGlashan TH, Grilo CM. Psychosocial impairment and treatment utilization by patients with borderline personality disorder, other personality disorders, mood and anxiety disorders, and a healthy comparison group. Compr Psychiatry. 2007; 48(4): 329-36.

5. Gunderson JG, Stout RL, McGlashan TH, Shea MT, Morey LC, Grilo CM, et al. Ten-year course of borderline personality disorder: psychopathology and function from

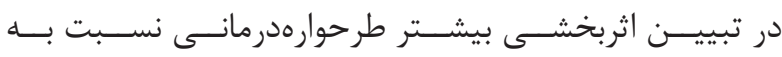

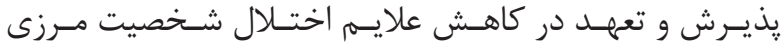

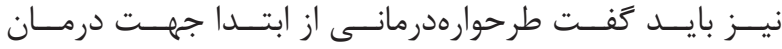

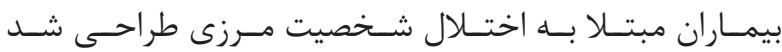

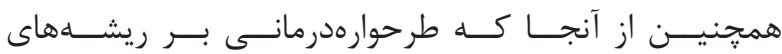

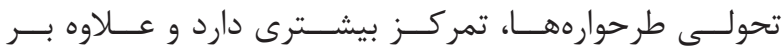

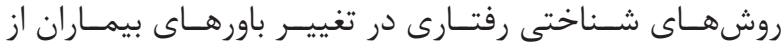

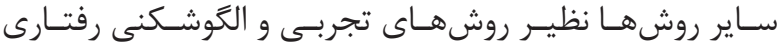

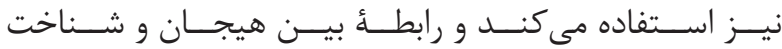

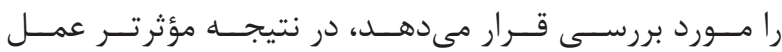
مى كنـــد.

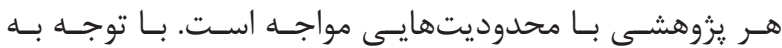

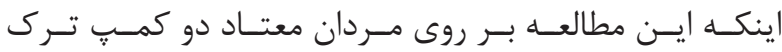

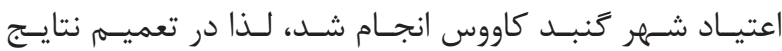

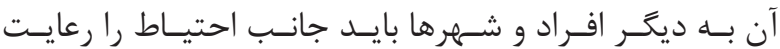

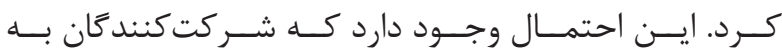

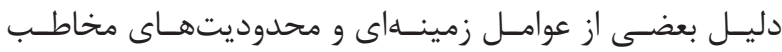

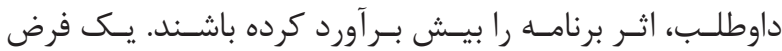

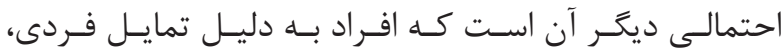

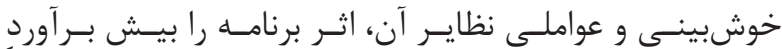

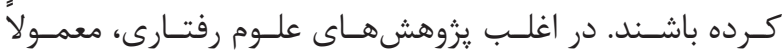

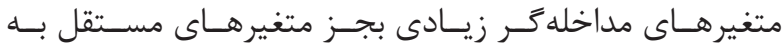

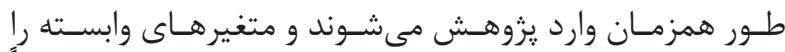

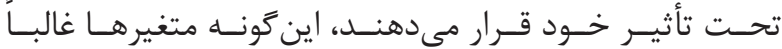

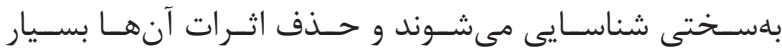

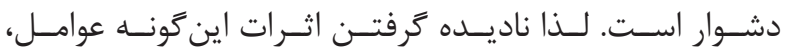

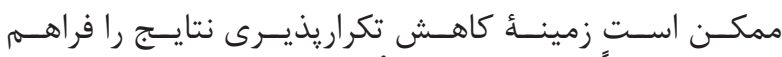

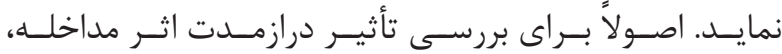

منابع

the collaborative longitudinal personality disorders study. Arch Gen Psychiatry. 2011; 68(8): 827-37.

6. Javaras KN, Zanarini MC, Hudson JI, Greenfield SF, Gunderson JG. Functional outcomes in communitybased adults with borderline personality disorder. J Psychiatr Res. 2017; 89: 105-14.

7. Skodol AE, Pagano ME, Bender DS, Shea MT, Gunderson JG, Yen S, et al. Stability of functional impairment in patients with schizotypal, borderline, avoidant, or obsessive-compulsive personality disorder over two years. Psychol Med. 2005; 35(3): 443-51.

8. Zanarini MC, Frankenburg FR, Reich DB, Fitzmaurice G. The 10-year course of psychosocial functioning among patients with borderline personality disorder and axis II comparison subjects. Acta Psychiatr Scand. 2010; 122(2): 103-9.

9. Van Asselt AD, Dirksen CD, Arntz A, Severens JL. The cost of borderline personality disorder: societal cost of illness in BPD-patients. Eur Psychiatry. 2007; 
22(6): 354-61.

10. Wagner T, Fydrich T, Stiglmayr C, Marschall P, Salize HJ, Renneberg B, et al. Societal cost-of-illness in patients with borderline personality disorder one year before, during and after dialectical behavior therapy in routine outpatient care. Behav Res Ther. 2014; 61: 12-22.

11. Ten Have M, Verheul R, Kaasenbrood A, van Dorsselaer S, Tuithof M, Kleinjan M, et al. Prevalence rates of borderline personality disorder symptoms: a study based on the Netherlands mental health survey and incidence Study-2. BMC Psychiatry. 2016; 16: 249. doi: 10.1186/s12888-016-0939-x.

12. Gunderson JG. Borderline personality disorder: ontogeny of a diagnosis. Am J Psychiatry. 2009; 166(5): 530-9.

13. Widiger TA, Weissman MM. Epidemiology of borderline personality disorder. Hosp Community Psychiatry. 1991; 42(10): 1015-21.

14. Dosti C, Gholami S, Toarabian S. The effectiveness of acceptance and commitment-based therapy on aggression reduction in students with internet addiction. Journal of Health Care. 2017; 2: 6.

15. Zanarini MC, Frankenburg FR, Reich DB, Silk KR, Hudson JI, McSweeney LB. The subsyndromal phenomenology of borderline personality disorder: a 10- year follow-up study. Am J Psychiatry. 2007; 164(6): 929-35.

16. Jacob GA, Guenzler C, Zimmermann S, Scheel $\mathrm{CN}$, Rüsch N, Leonhart R, et al. Time course of anger and other emotions in women with borderline personality disorder: a preliminary study. J Behav Ther Exp Psychiatry. 2008; 39(3): 391-402.

17. Rüsch N, Lieb K, Göttler I, Hermann C, Schramm E, Richter H, et al. Shame and implicit self-concept in women with borderline personality disorder. Am J Psychiatry. 2007; 164(3): 500-8.

18. Gratz KL, Rosenthal MZ, Tull MT, Lejuez CW, Gunderson JG. An experimental investigation of emotional reactivity and delayed emotional recovery in borderline personality disorder: the role of shame. Compr Psychiatry. 2010; 51(3): 275-85.

19. Stiglmayr CE, Grathwol T, Linehan MM, Ihorst G, Fahrenberg J, Bohus M. Aversive tension in patients with borderline personality disorder: a computer-based controlled field study. Acta Psychiatr Scand. 2005; 111(5): 372-9.

20. Arntz A, Klokman J, Sieswerda S. An experimental test of the schema mode model of borderline personality disorder. J Behav Ther Exp Psychiatry. 2005; 36(3): 226-39.

21. Links PS, Heslegrave RJ. Prospective studies of outcome. Understanding mechanisms of change in patients with borderline personality disorder. Psychiatr Clin North Am. 2000; 23(1): 137-50.

22. Choi-Kain LW, Finch EF, Masland SR, Jenkins JA, Unruh BT. What works in the treatment of borderline personality disorder? Curr Behav Neurosci Rep. 2017; 4(1): 21-30.

23. Stoffers JM, Vollm BA, Rucker G, Timmer A, Huband N, Lieb K. Psychological therapies for people with borderline personality disorder. Cochrane Database Syst Rev. 2012; (8): CD005652. doi: 10.1002/14651858. CD005652.pub2.

24. Links PS, Shah R, Eynan R. Psychotherapy for borderline personality disorder: Progress and remaining challenges. Curr Psychiatry Rep. 2017; 19(3): 16. doi: 10.1007/s11920-017-0766-x.

25. Young JE, Klosko JS, Weishaar ME. Schema therapy: A practioner's guide. $1^{\text {st }}$ ed. New York: Guilford Press; 2003.

26. Vyskocilova J, Prasko J, Sedlackova Z, Ociskova M, Grambal A. Schema therapy for CBT therapists who treat borderline patients. Act Nerv Super Rediviva. 2014; 56(1-2): 24-31.

27. Jenkins G. An investigation of schema modes in the eating disordered population.) dissertation (. The University of Edinburgh; 2009.

28. Renner F, Lobbestael J, Peeters F, Arntz A, Huibers M. Early maladaptive schemas in depressed patients: stability and relation with depressive symptoms over the course of treatment. J Affect Disord. 2012; 136(3): 581-90.

29. Sempértegui GA, Karreman A, Arntz A, Bekker MH. Schema therapy for borderline personality disorder: a comprehensive review of its empirical foundations, effectiveness and implementation possibilities. Clin Psychol Rev. 2013; 33(3): 426-47.

30. Lobbestael J, van Vreeswijk M, Spinhoven P, Schouten E, Arntz A. Reliability and validity of the short Schema Mode Inventory (SMI). Behav Cogn Psychother. 2010; 38(4): 437-58.

31. Tan YM, Lee CW, Averbeck LE, Brand-de Wilde O, Farrell J, Fassbinder E, et al. Schema therapy for borderline personality disorder: A qualitative study 
of patients' perceptions. PLoS One. 2018; 13(11): e0206039.

32. Jacob GA, Hauer A, Köhne S, Assmann N, Schaich A, Schweiger U, et al. A schema therapy-based ehealth program for patients with borderline personality disorder (priovi): Naturalistic single-arm observational study. JMIR Ment Health. 2018; 5(4): e10983.

33. Wetzelaer P, Farrell J, Evers SM, Jacob GA, Lee $\mathrm{CW}$, Brand $\mathrm{O}$, et al. Design of an international multicentre RCT on group schema therapy for borderline personality disorder. BMC Psychiatry. 2014; 14: 319. doi.org/10.1186/s12888-014-0319-3.

34. Dickhaut V, Arntz A. Combined group and individual schema therapy for borderline personality disorder: a pilot study. J Behav Ther Exp Psychiatry. 2014; 45(2): 242-51.

35. Giesen-Bloo J, van Dyck R, Spinhoven $\mathrm{P}$, van Tilburg W, Dirksen C, van Asselt T, et al. Outpatient psychotherapy for borderline personality disorder: randomized trial of schema-focused therapy vs transference-focused psychotherapy. Arch Gen Psychiatry. 2006; 63(6): 649-58.

36. Nadort M, Arntz A, Smit JH, Giesen-Bloo J, Eikelenboom M, Spinhoven P, et al. Implementation of outpatient schema therapy for borderline personality disorder: study design. BMC Psychiatry. 2009; 9: 64. doi: $\quad 10.1186 / 1471-244 X-9-64$.

37. Peymannia B, Hamid N, Mahmoud Alilo, M. The effectiveness of compassionate acceptance and commitment therapy matrix on self-harmful behaviors and quality of life in students with symptoms of borderline personality disorder. Psychological Achievement. 2019; 25(1): 23-44.

38. Bricker J, Tollison, S. Comparison of motivational interviewing with acceptance and commitment therapy: A conceptual and clinical review. Behav Cogn Psychother. 2011; 39(5): 541-59.

39. Hayes SC, Strosahl KD, Bunting K, Twohig M, Wilson KG. What is acceptance and commitment therapy? SC. Hayes, KD Strosahl. A practical guide to acceptance and commitment therapy. New York, NY: Springer Science-Business Media. 2010; p. 3-29.

40. Roditi D, Robinson ME. The role of psychological interventions in the management of patients with chronic pain. Psychol Res Behav Manag. 2011; 4: 41-9.

41. Twohig MP, Hayes SC, Masuda A. Increasing willingness to experience obsessions: Acceptance and commitment therapy as a treatment for obsessive compulsive disorder. Behav Ther. 2006; 37(1): 3-13.
42. Chakhssi F, Janssen W, Pol SM, van Dreumel M, Westerhof GJ. Acceptance and commitment therapy group-treatment for non-responsive patients with personality disorders: An exploratory study. Personal Ment Health. 2015; 9(4): 345-56.

43. Yardley J. Treatment of pediatric obsessivecompulsive disorder: utilizing parent-facilitated acceptance and commitment therapy. [doctoral dissertation]. [Logan, USA]: Faculty of Psychology, Utah State University. 2012; p. 74-7.

44. Twohig MP, Hayes SC, Plumb JC, Pruitt LD, Collins AB, Hazlett-Stevens $H$, et al. A randomized clinical trial of acceptance and commitment therapy versus progressive relaxation training for obsessive-compulsive disorder. J Consult Clin Psychol. 2010; 78(5): 705-16.

45. Craske MG, Niles AN, Burklund LJ, WolitzkyTaylor KB, Vilardaga JC, Arch JJ4, et al. Randomized controlled trial of cognitive behavioral therapy and acceptance and commitment therapy for social phobia: outcomes and moderators. J Consult Clin Psychol. 2014; 82(6): 1034-48.

46. Hichem M, Figen S, Timurab C. Novel technologies in detection, treatment and prevention of substance use disorders. Journal of Food and Drug Analysis. 2019; 27(1): 22-31.

47. Forman EM, Herbert JD, Moitra E, Yeomans PD, Geller PA. A randomized controlled effectiveness trial of acceptance and commitment therapy and cognitive therapy for anxiety and depression. Behavior Modification. 2007; 31(6): 772-99.

48. Arch JJ, Eifert GH, Davies C, Plumb Vilardaga JC, Rose RD, Craske MG. Randomized clinical trial of cognitive behavioral therapy (CBT) versus acceptance and commitment therapy (ACT) for mixed anxiety disorders. J Consult Clin Psychol. 2012; 80(5): 750-65.

49. Morton J, Snowdon S, Gopold M, Guymer E. Acceptance and commitment therapy group treatment for symptoms of borderline personality disorder: A public sector pilot study. Cogn Behav Pract. 2012; 19: 527-44.

50. World Medical A. World medical association declaration of helsinki: ethical principles for medical research involving human subjects. JAMA. 2013; 310(20): 2191-4.

51. Arntz A, Van Genderen H. Schema therapy for borderline personality disorder. NewYork: John Wiley \& Sons; 2009.

52. Bigdeli I, Rezaei AM, Arab Z. Study of subtypes of ASPD and comorbidity with anxiety and opioid disorders. Journal of Clinical Psychology. 2013; 5(1): 1-9. 
53. Debashi L, Najafi M, Rahimian-Boogar I. The effectiveness of cognitive analytic therapy on impulsivity and dissociative experiences of borderline personality disordered patients. Journal of Clinical Psychology. 2018; 10(2): 15-27.

54. Sharifi AA, Molavi H, Namdari C. Triple validity multi-axis clinical trial milne-3. Journal of Applied Psychology. 2007; 9(34): 27-38.

55. Lobbestael J, Van Vreeswijk MF, Arntz A. An empirical test of schema mode conceptualizations in personality disorders. Behav Res Ther. 2008; 46(7): 854-60.

56. Mohamadizadeh L, Makvandi B, Pasha R, BakhtiarPour S, Hafezi F. Compare the efficacy of dialectical behavior therapy (DBT) and schema therapy (ST) on impulsive behavior in patients with borderline personality disorder. Journal of Guilan University of Medical Sciences. 2018; 27(106): 44-53.

57. Farrell JM, Shaw IA, Webber MA. A schema-focused approach to group psychotherapy for outpatients with borderline personality disorder: a randomized controlled trial. J Behav Ther Exp Psychiatry. 2009; 40(2): 317-28.

58. Fassbinder E, Schuetze M, Kranich A, Sipos V, Hohagen F, Shaw I, et al. Feasibility of group schema therapy for outpatients with severe borderline personality disorder in Germany: A pilot study with three-year follow-up. Front Psychol. 2016; 7: 1851. eCollection. doi: $10.3389 /$ fpsyg.2016.01851.

59. Gratz KL, Gunderson JG. Preliminary data on an acceptance-based emotion regulation group intervention for deliberate self-harm among women with borderline personality disorder. Behav Ther. 2006; 37(1): 25-35.

60. Sharp C, Kalpakci A, Mellick W, Venta A, Temple
JR. First evidence of a prospective relation between avoidance of internal states and borderline personality disorder features in adolescents. Eur Child Adolesc Psychiatry. 2015; 24(3): 283-90.

61. Twohig MP. Introduction: The basics of acceptance and commitment therapy. Cogn Behav Pract. 2012; 19: 499-507.

62. Chapman AL, Gratz KL, Brown MZ. Solving the puzzle of deliberate self-harm: The experiential avoidance model. Behav Res Ther. 2006; 44(3): 371-94.

63. Chapman AL, Specht MW, Cellucci T. Borderline personality disorder and deliberate self-harm: does experiential avoidance play a role? Suicide Life Threat Behav. 2005; 35(4): 388-99.

64. Iverson KM, Follette VM, Pistorello J, Fruzzetti AE. An investigation of experiential avoidance, emotion dysregulation, and distress tolerance in young adult outpatients with borderline personality disorder symptoms. Personal Disord. 2012; 3(4): 415-22.

65. Berking M, Neacsiu A, Comtois KA, Linehan MM. The impact of experiential avoidance on the reduction of depression in treatment for borderline personality disorder. Behav Res Ther. 2009; 47(8): 663-70.

66. Polk KL, Schoendorff B, Webster M, Olaz F. The essential guide to the ACT matrix. context press, An Imprint of New Harbinger Publications, Inc. Oakland, CA; 2016.

67. Razzaque R. An acceptance and commitment therapy-based protocol for the management of acute selfharm and violence in severe mental illness. Journal of Psychiatric Intensive Care. 2013; 9(2): 72-6. 